University of Parma Preprint UPRF-95-438

December 1995

\title{
Gauging kinematical and internal symmetry groups for extended systems: the Galilean one-time and two-times harmonic oscillators *
}

\author{
R. De Pietri†, L. Lusannał and M. Pauri†† \\ †Dipartimento di Fisica - Sezione di Fisica Teorica, Università di Parma, 43100 \\ Parma, Italy and I.N.F.N., Sezione di Milano, Gruppo Collegato di Parma. \\ $\ddagger I . N . F . N$., Sezione di Firenze, Largo E. Fermi 2, Arcetri - 50127 Firenze - Italy. \\ $\dagger \dagger$ Center for Philosophy of Science, University of Pittsburgh, Pittsburgh PA, USA. $\sharp$
}

\begin{abstract}
The possible external couplings of an extended non-relativistic classical system are characterized by gauging its maximal dynamical symmetry group at the center-of-mass. The Galilean one-time and two-times harmonic oscillators are exploited as models. The following remarkable results are then obtained: 1) a peculiar form of interaction of the system as a whole with the external gauge fields; 2) a modification of the dynamical part of the symmetry transformations, which is needed to take into account the alteration of the dynamics itself, induced by the gauge fields. In particular, the Yang-Mills fields associated to the internal rotations have the effect of modifying the time derivative of the internal variables in a scheme of minimal coupling (introduction of an internal covariant derivative); 3) given their dynamical effect, the Yang-Mills fields associated to the internal rotations apparently define a sort of Galilean spin connection, while the Yang-Mills fields associated to the quadrupole momentum and to the internal energy have the effect of introducing a sort of dynamically induced internal metric in the relative space.
\end{abstract}

PACS numbers: 04.90.+e, 04.20.Fy, 03.20.+i, 11.15.-9, 11.30.-j, 11.30.Ly

Short title: Gauging kinematical and internal dynamical symmetries.

August 28, 2021

\footnotetext{
* This work has been supported partly by I.N.F.N., Italy (iniziativa specifica FI-2) and partly by the Network Constrained Dynamical Systems of the E.U. Programme "Human Capital and Mobility". $\sharp$ On leave from Dipartimento di Fisica - Sezione di Fisica Teorica, Università di Parma, 43100 Parma, Italy and I.N.F.N., Sezione di Milano, Gruppo Collegato di Parma.
} 


\section{Introduction}

In this work we exploit a gauge technique introduced in previous papers [1] to characterize the possible external couplings of extended non-relativistic dynamical systems. The non-relativistic isotropic harmonic oscillator with center-of-mass is used as a model both in the standard Newtonian description and in the presymplectic description with two first-class constraints.

As well known, no natural prescription is available for carrying out the coupling to external fields of an extended system with certain conserved charges [mass, internal energy, intrinsic angular momentum (invariants of the extended Galilei group $\dagger$ ), quadrupole momentum for the oscillator, Runge-Lenz vector for the Kepler system, and so on). Intuitively, one would expect that e.g. a gravitational field would probe the individual constituent masses (as it happens for a dust of free particles); it is not clear, however, what to do in case the constituents of the system are not free (cluster of interacting particles).

Since the only constructive and self-consistent method to create a coupling to external fields, in the case in which the conserved charges are connected to a Lie algebra ( $1^{\text {st }}$ Noether theorem), is the Utiyama procedure, in this work we make the ansatz that the essential structural elements of the spatial extension of a dynamical system are represented and summarized by its maximal dynamical symmetry group viz. by the algebraic structure of the whole set of the constants of the motion of the system. This seems to be a natural development and enrichment of the tratidional "atomistic" way of describing extended systems in terms of constituent parts interacting among themselves.

The maximal dynamical symmetry group of the isotropic harmonic oscillator with center-of-mass is the semi-direct product of the centrally extended Galilei group and the $S U(3) \otimes U(1)$ group. Actually, the explicit canonical realization $\ddagger$ of this maximal group is the direct product of a singular realization of the $S U(3) \otimes U(1)$ group and the singular scalar realization of the centrally extended Galilei group, corresponding to the center-of-mass of the system [6].

Thus, given the peculiar algebraic structure of the realization of the maximal dynamical symmetry, we are led to implement our ansatz by localizing the gauging of the symmetry at the center-of-mass of the system. We show thereby that the gauge procedure is meaningful also for dynamical symmetries besides the usual kinematical ones.

Let us remark that our ansatz seems suited for clusters of interacting particles both in the case of confining forces and of long-range interactions. A priori, this algebraic ansatz could be applied also to a dust of free particles; this, however, would result in a non-standard coupling of the external fields to the center-of-mass and relative variables of the dust. It seems realistic to retain the standard coupling to the dust, but use our ansatz for the constituents of a dust of extended bound systems (for instance a dust of harmonic oscillators).

In spite of the evident paradigmatic and heuristic nature of our ansatz, the results obtained here seem to be notably expressive.

The technical steps of the work are the following: 1) the standard Utiyama

$\dagger$ Note that, on the one hand, all the massive systems are realizations of the extended Galilei group, and, on the other, as shown in our previous papers [1], recourse to the extended group is a necessary condition to implement the Utiyama procedure.

$\ddagger$ see Section 2 and the Appendices. 
procedure for fields is applied to the possible trajectories of the center-of-mass of the system as described by a canonical realization of the extended Galilei group. This determines the gravitational-inertial fields which can couple to the center-ofmass itself. As shown elsewhere [1], the requirement of invariance (properly quasiinvariance) of the Lagrangian leads to the introduction of eleven gauge compensating fields and their transformation properties. 2) The generalized Utiyama procedure is then applied to the internal dynamical $S U(3) \otimes U(1)$ symmetry so that gauge compensating fields have to be introduced in connection to the internal angular momentum (spin), the quadrupole momentum and the internal energy. As already indicated by the above structure, the crucial point is that, not only the internal transformations have a dynamical nature but, also, that the Galilei rotations subgroup affects the internal transformations so that a semi-direct product structure emerges.

The following remarkable results are then obtained: 1) a peculiar form of interaction of the system as a whole with the external gauge fields; 2) a modification of the dynamical part of the symmetry transformations, which is needed to take into account the modification of the dynamics itself induced by the gauge fields. In

particular, the Yang-Mills fields associated to the internal rotations have the effect of modifying the time derivative of the internal variables in a scheme of minimal coupling (introduction of an internal covariant derivative). Moreover, it is interesting to find that, given their dynamical effect, the Yang-Mills fields associated to the internal rotations apparently define a sort of Galilean spin connection. On the other hand, the Yang-Mills fields associated to the quadrupole momentum and to the internal energy have the effect of introducing a sort of dynamically induced internal metric in the relative space, together with the above mentioned modification of the interaction.

These general features are reminiscent of some peculiar aspects of the relativistic string dynamics, in particular of the so called bootstrap hypothesis, according to which the external fields that can couple to the system are all and the same which are already included in the field theory itself. Of course, the maximal symmetry group of the relativistic string is not a Lie group but it is based instead on the infinite algebra of the constants of motion studied by Pohlmeyer and Rehren [5]. The specific study of this dynamical system will require a preliminary clarification of the case of the relativistic oscillator, in which a simple separation of the symmetry into centerof-mass and internal parts is no longer feasible. Finally, the determination of the dynamical equations for the Yang-Mills fields remains an open problem and will be discussed elsewhere.

\section{The one-time harmonic oscillator with center-of-mass}

The Lagrangian that describe the one-time harmonic oscillator with center-of-mass is:

$$
\mathcal{L}=\sum_{a=1}^{2} \frac{m_{a}}{2} \delta_{i j} \dot{x}_{a}^{i} \dot{x}_{a}^{j}-\frac{k}{2} \delta_{i j}\left(x_{1}^{i}-x_{2}^{i}\right)\left(x_{1}^{j}-x_{2}^{j}\right) .
$$

Introducing the center-of-mass and relative coordinates:

$$
\left\{\begin{array}{l}
x^{i}=\frac{m_{1} x_{1}^{i}+m_{2} x_{2}^{i}}{M} \\
r^{i}=x_{1}^{i}-x_{2}^{i}
\end{array}\right.
$$


the Lagrangian can be rewritten as:

$$
\mathcal{L}=\frac{M}{2} \delta_{i j} \dot{x}^{i} \dot{x}^{j}+\frac{\mu}{2} \delta_{i j} \dot{r}^{i} \dot{r}^{j}-\frac{k}{2} \delta_{i j} r^{i} r^{j} \stackrel{\text { def }}{=} \frac{M}{2} \delta_{i j} \dot{x}^{i} \dot{x}^{j}+\mathcal{L}_{r}(t),
$$

where $\mu=\frac{m_{1} m_{2}}{m_{1}+m_{2}}$ is the reduced-mass and $M=m_{1}+m_{2}$ is the total mass.

The canonical conjugate momenta are:

$$
\begin{cases}p_{1 i} & \stackrel{\text { def }}{=} \frac{\partial L}{\partial \dot{x}_{1}^{i}}=m_{1} \delta_{i j} \dot{x}_{1}^{j}=\frac{m_{1}}{M} P_{i}+\pi_{i} \\ p_{2 i} & \stackrel{\text { def }}{=} \frac{\partial L}{\partial \dot{x}_{2}^{i}}=m_{2} \delta_{i j} \dot{x}_{2}^{j}=\frac{m_{2}}{M} P_{i}-\pi_{i}\end{cases}
$$

where

$$
\left\{\begin{aligned}
P_{i} & \stackrel{\text { def }}{=} \frac{\partial L}{\partial \dot{x}^{i}}=M \delta_{i j} \dot{x}^{j}=p_{1 i}+p_{2 i} \\
\pi_{i} & \stackrel{\text { def }}{=} \frac{\partial L}{\partial \dot{r}^{i}}=\mu \delta_{i j} \dot{r}^{j}=\frac{m_{2} p_{1 i}-m_{1} p_{2}}{M}
\end{aligned}\right.
$$

In phase space we have the following non-zero Poisson brackets:

$$
\left\{\begin{array}{rlll}
\left\{x_{1}^{i}, p_{1 j}\right\} & =\left\{x_{2}^{i}, p_{2 j}\right\}=\delta_{j}^{i} & a, b=1,2,3 \\
\left\{x^{i}, P_{j}\right\} & =\left\{r^{a}, \pi_{b}\right\}=\delta_{j}^{i} & i, j=1,2,3
\end{array}\right.
$$

and the Hamiltonian results

$$
\left\{\begin{aligned}
\bar{H} & =\frac{1}{2 m_{1}} \delta^{i j} p_{1 i} p_{1 j}+\frac{1}{2 m_{2}} \delta^{i j} p_{2 i} p_{2 j}+\frac{k}{2} \delta_{i j}\left(x_{1}^{i}-x_{2}^{i}\right)\left(x_{1}^{j}-x_{2}^{j}\right) \\
& =\frac{1}{2 M} \delta^{i j} P_{i} P_{j}+\overline{\mathcal{E}} \\
\overline{\mathcal{E}} & \stackrel{\text { def }}{=} \frac{1}{2 \mu} \delta^{a b} \pi_{a} \pi_{b}+\frac{k}{2} \delta_{a b} r^{a} r^{b}
\end{aligned}\right.
$$

where $\overline{\mathcal{E}}$ is the internal energy (from now on, we shall use the indexes $i, j, k,$. for the center-of-mass components and $a, b, c, d, \ldots$ for the internal components). The notation $\bar{f}$ denotes a function on phase-space and the symbol $\stackrel{!}{=}$ the pull-back via the Legendre transformation. i.e. $\bar{f}(\boldsymbol{x}, \boldsymbol{P} ; \boldsymbol{r}, \boldsymbol{\pi}) \stackrel{!}{=} f(\boldsymbol{x}, \dot{\boldsymbol{x}} ; \boldsymbol{r}, \dot{\boldsymbol{r}})$. Before proceeding, let us fix some notations in connection with more general classes of functions that will have to be considered. Precisely: 1) $f(\boldsymbol{z}, t)$, with $\boldsymbol{z}$ and $t$ independent variables; and 2) $f(\boldsymbol{x}(t), t)$. by

The variations in case 1 ), when $t \rightarrow t^{*}=t+\delta t, \boldsymbol{z} \rightarrow \boldsymbol{z}^{*}=\boldsymbol{z}+\delta \boldsymbol{z}$, will be denoted

$$
\begin{aligned}
\delta f(\boldsymbol{z}, t) & =f^{*}\left(\boldsymbol{z}^{*}, t^{*}\right)-f(\boldsymbol{z}, t) \\
\delta_{0} f(\boldsymbol{z}, t) & =f^{*}(\boldsymbol{z}, t)-f(\boldsymbol{z}, t)= \\
& =\delta f(\boldsymbol{z}, t)-\frac{\partial f(\boldsymbol{z}, t)}{\partial z^{k}} \delta z^{k}-\frac{\partial f(\boldsymbol{z}, t)}{\partial t} \delta t .
\end{aligned}
$$

On the other hand, in case 2), it is convenient to distinguish three kinds of variations. Precisely, in correspondence to:

$$
\begin{cases}t & \rightarrow t^{*}=t+\delta t \\ \boldsymbol{x}(t) & \rightarrow \boldsymbol{x}^{*}\left(t^{*}\right)=\boldsymbol{x}(t)+\delta \boldsymbol{x}(t)=\boldsymbol{x}(t)+\delta_{0} \boldsymbol{x}(t)+\frac{d \boldsymbol{x}(t)}{d t} \delta t\end{cases}
$$


we shall define:

$$
\begin{aligned}
\delta f(\boldsymbol{x}(t), t) & =f^{*}\left(\boldsymbol{x}^{*}\left(t^{*}\right), t^{*}\right)-f(\boldsymbol{x}(t), t) \\
\delta_{0} f(\boldsymbol{x}(t), t) & =f^{*}(\boldsymbol{x}(t), t)-f(\boldsymbol{x}(t), t)= \\
& =\delta f(\boldsymbol{x}(t), t)-\frac{\partial f(\boldsymbol{x}(t), t)}{\partial x^{k}} \delta x^{k}(t)-\frac{\partial f(\boldsymbol{x}(t), t)}{\partial t} \delta t \\
\delta_{0[t]} f(\boldsymbol{x}(t), t) & =f^{*}\left(\boldsymbol{x}^{*}(t), t\right)-f(\boldsymbol{x}(t), t)= \\
& =\delta f(\boldsymbol{x}(t), t)-\left[\frac{\partial f(\boldsymbol{x}(t), t)}{\partial x^{k}} \frac{d x^{k}(t)}{d t}+\frac{\partial f(\boldsymbol{x}(t), t)}{\partial t}\right] \delta t .
\end{aligned}
$$

The generators of the Galilei group $\mathcal{G}$ for the particular realization of the harmonic oscillator can be written $\left(c_{i j}{ }^{k}=\epsilon^{i j k}\right.$ structure constants of the $\mathrm{SU}(2)$ group):

$$
\left\{\begin{aligned}
\bar{H} & =\frac{\delta^{i j} P_{i} P_{j}}{2 M}+\overline{\mathcal{E}} \\
P_{i} & =p_{1 i}+p_{2 i} \\
\bar{J}_{i} & =c_{i j}{ }^{k} x_{1}^{j} p_{1 k}+c_{i j}{ }^{k} x_{2}^{j} p_{2 k} \\
\bar{K}_{i} & =m_{1} \delta_{i j} x_{1}^{j}+m_{2} \delta_{i j} x_{2}^{j}-t\left(p_{1 i}+p_{2 i}\right)
\end{aligned}\right.
$$

and they satisfy the centrally extended Galilei algebra with central charge $M$,

$$
\left\{\begin{aligned}
\left\{\bar{H}, \bar{K}_{i}\right\} & =-P_{i} \\
\left\{P_{i}, \bar{K}_{j}\right\} & =-M \delta_{i j} \\
\left\{P_{i}, \bar{J}_{j}\right\} & =c_{i j}{ }^{k} P_{k} \\
\left\{\bar{J}_{i}, \bar{J}_{j}\right\} & =c_{i j}{ }^{k} \bar{J}_{k} \\
\left\{\bar{K}_{i}, \bar{J}_{j}\right\} & =c_{i j}{ }^{k} \bar{K}_{k} \\
\left\{\bar{K}_{i}, \bar{K}_{j}\right\} & =0
\end{aligned}\right.
$$

The generator of a general Galilei transformation is then

$$
\bar{G}=\varepsilon \bar{H}+\varepsilon^{i} P_{i}+\omega^{i} \bar{J}_{i}+v^{i} \bar{K}_{i}
$$

and the induced coordinate transformations are

$$
\left\{\begin{array}{l}
\bar{\delta}_{0} x^{i}=\left\{x^{i}, \bar{G}\right\}=\epsilon \delta^{i j} \frac{P_{j}}{M}+\epsilon^{i}+c_{j k}{ }^{i} \omega^{j} x^{k}-t v^{i} \\
\bar{\delta}_{0} r^{a}=\left\{r^{a}, \bar{G}\right\}=\epsilon \delta^{a b} \frac{\pi_{b}}{\mu}+c_{b c}{ }^{a} \omega^{b} r^{c} \\
\bar{\delta}_{0} x_{1}^{i}=\left\{x_{1}^{i}, \bar{G}\right\}=\epsilon \delta^{i j} \frac{p_{1 j}}{m_{1}}+\epsilon^{i}+c_{j k}{ }^{i} \omega^{j} x_{1}^{k}-t v^{i} \\
\bar{\delta}_{0} x_{2}^{i}=\left\{x_{2}^{i}, \bar{G}\right\}=\epsilon \delta^{i j} \frac{p_{2 j}}{m_{2}}+\epsilon^{i}+c_{j k}{ }^{i} \omega^{j} x_{2}^{k}-t v^{i}
\end{array}\right.
$$

$\bar{G}$ gives rise to the following Noether transformations

$$
\left\{\begin{array}{l}
\delta_{0} x^{i}=\epsilon \dot{x}^{i}+\epsilon^{i}+c_{j k}{ }^{i} \omega^{j} x^{k}-t v^{i} \\
\delta_{0} r^{a}=\epsilon \dot{r}^{a}+c_{c d}{ }^{a} \omega^{b} r^{c} \\
\delta_{0} x_{1}^{i}=\epsilon \dot{x}_{1}^{i}+\epsilon^{i}+c_{j k}{ }^{i} \omega^{j} x_{1}^{k}-t v^{i} \\
\delta_{0} x_{2}^{i}=\epsilon \dot{x}_{2}^{i}+\epsilon^{i}+c_{j k} \omega^{j} x_{2}^{k}-t v^{i},
\end{array}\right.
$$


under which the Lagrangian is quasi-invariant. In fact:

$$
\begin{aligned}
\delta_{0} \mathcal{L} & =\frac{\partial \mathcal{L}}{\partial x^{i}} \delta x^{i}+\frac{\partial \mathcal{L}}{\partial r^{a}} \delta r^{a}+\frac{\partial \mathcal{L}}{\partial \dot{x}^{i}} \frac{d}{d t} \delta x^{i}+\frac{\partial \mathcal{L}}{\partial \dot{r}^{a}} \frac{d}{d t} \delta r^{a} \\
& =\frac{d}{d t}\left[\epsilon \mathcal{L}-M \delta_{i j} v^{i} x^{j}\right] .
\end{aligned}
$$

Therefore, the Galilei generators are constants of the motion and the Galilei group is a kinematical symmetry group of the system.

As well known, in the case of a harmonic oscillator, we have an additional dynamical symmetry group, viz. $S U(3) \otimes U(1)$, whose real form realization has the following generators

$$
\left\{\begin{aligned}
\overline{\mathcal{E}}^{\prime} & =\sqrt{\frac{\mu}{k}} \overline{\mathcal{E}}=\frac{1}{2}\left(\frac{1}{\sqrt{\mu k}} \delta^{a b} \pi_{a} \pi_{b}+\sqrt{\mu k} \delta_{a b} r^{a} r^{b}\right) \\
& \stackrel{!}{=} \frac{1}{2}\left(\frac{\mu^{2}}{\sqrt{\mu k}} \delta_{a b} \dot{r}^{a} \dot{r}^{b}+\sqrt{\mu k} \delta_{a b} r^{a} r^{b}\right) \\
\bar{S}_{a} & =(\vec{r} \wedge \vec{\pi})_{a}=c_{a b}{ }^{c} r^{b} \pi_{c} \stackrel{!}{=} \mu c_{a b}{ }^{c} r^{b} \delta_{c c_{1}} \dot{r}^{c_{1}} \\
\overline{\mathcal{Q}}_{a b} & =\frac{1}{2 \sqrt{\mu k}} \pi_{a} \pi_{b}+\frac{\sqrt{\mu k}}{2} \delta_{a c} \delta_{b d} r^{c} r^{d}-\delta_{a b} \frac{\overline{\mathcal{E}}^{\prime}}{3} \\
& \stackrel{!}{=} \delta_{a c} \delta_{b d}\left[\frac{\mu^{2}}{2 \sqrt{\mu k}} \dot{r}^{c} \dot{r}^{d}+\frac{\sqrt{\mu k}}{2} r^{c} r^{d}\right]-\delta_{a b} \frac{\mathcal{E}^{\prime}}{3}
\end{aligned}\right.
$$

Here $\overline{\mathcal{E}}$ is the internal energy, $\bar{S}_{a}$ the internal angular momentum ("spin") and $\overline{\mathcal{Q}}_{a b}$ the quadrupole moment. $\overline{\mathcal{E}}$ and $\overline{\mathcal{Q}}_{a b}$ may be described in a more compact form by the symmetric tensor

$$
\begin{aligned}
\bar{N}_{a b} & =\frac{1}{2 \sqrt{\mu k}} \pi_{a} \pi_{b}+\frac{\sqrt{\mu k}}{2} \delta_{a c} \delta_{b d} r^{c} r^{d} \\
& =\overline{\mathcal{Q}}_{a b}+\delta_{a b} \frac{\overline{\mathcal{E}}^{\prime}}{3} .
\end{aligned}
$$

A real form of the $S U(3) \otimes U(1)$ algebra is

$$
\left\{\begin{array}{rl}
\left\{\bar{S}_{a}, \bar{S}_{b}\right\} & =c_{a b}{ }^{c} \bar{S}_{c} \\
\left\{\bar{S}_{a}, \bar{N}_{c d}\right\} & =c_{a c}{ }^{b} \bar{N}_{b d}+c_{a d}{ }^{b} \bar{N}_{c b} \\
\left\{\bar{N}_{a b}, \bar{N}_{c d}\right\} & =\frac{1}{4}\left[\delta_{b d} c_{a c} e+\delta_{b c} c_{a d}{ }^{e}+\delta_{a d} c_{b c}{ }^{e}+\delta_{a c} c_{b d}{ }^{e}\right] \bar{S}_{e}
\end{array} .\right.
$$

Some other properties of the canonical $S U(3) \otimes U(1)$ realizations are reported in Appendix A.

The general $S U(3) \otimes U(1)$ transformations generated by $\tilde{G} \stackrel{\text { def }}{=} \theta^{a} \bar{S}_{a}+\xi^{\prime a b} \overline{\mathcal{Q}}_{a b}+\xi^{\prime} \mathcal{E}^{\prime}$ or equivalently

$$
\tilde{G} \stackrel{\text { def }}{=} \theta^{a} \bar{S}_{a}+\xi^{a b} \bar{N}_{a b}
$$

induce the following Noether transformations

$$
\left\{\begin{array}{l}
\bar{\delta}_{0} x^{i} \stackrel{!}{=} \delta_{0} x^{i}=0 \\
\bar{\delta}_{0} r^{a} \stackrel{!}{=} \delta_{0} r^{a}=c_{b c}{ }^{a} \theta^{b} r^{c}+\sqrt{\frac{\mu}{k}} \xi^{a b} \delta_{b c} \dot{r}^{c}
\end{array}\right.
$$


under which the Lagrangian is quasi-invariant:

$$
\begin{aligned}
\delta_{0} \mathcal{L} & =\frac{\partial \mathcal{L}}{\partial r^{a}} \delta_{0} r^{a}+\frac{\partial \mathcal{L}}{\partial r^{a}} \frac{d}{d t} \delta_{0} r^{a}=\frac{d}{d t}\left[\sqrt{\frac{\mu}{k}} \xi^{a b} \delta_{a c} \delta_{b d}\left(\frac{\mu}{2} \dot{r}^{c} \dot{r}^{d}-\frac{k}{2} r^{c} r^{d}\right)\right] \\
& \equiv \frac{d}{d t}\left[\sqrt{\frac{\mu}{k}} \xi^{a b} F_{a b}(\dot{\boldsymbol{r}} ; \boldsymbol{r})\right] .
\end{aligned}
$$

Therefore the $S U(3) \otimes U(1)$ generators are constants of the motion too.

Under the transformations (2.21), the generators $\bar{S}_{a}, \bar{N}_{a b}$ behave in the following way:

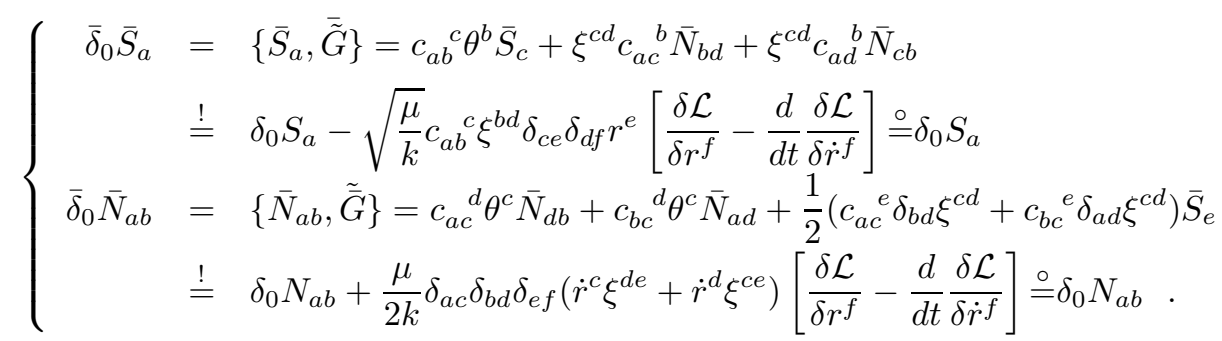

By "putting together" the central extension of the Galilei algebra and the $S U(3) \otimes U(1)$ algebra, we obtain the Lie algebra $\mathcal{G}_{\mathcal{H}}$ of the maximal symmetry group $\mathcal{H}$ whose Poisson brackets are given by eqs (2.12), (2.19) and

$$
\left\{\begin{array}{rl}
\left\{\bar{S}_{a}, \bar{J}_{i}\right\} & =\epsilon_{a i c} \bar{S}_{c} \\
\left\{\bar{N}_{a b}, \bar{J}_{i}\right\} & =\epsilon_{a i c} \bar{N}_{c b}+\epsilon_{b i c} \bar{N}_{a c}
\end{array} .\right.
$$

We get in this way a semi-direct product structure for the maximal symmetry group: $\mathcal{H}=\left(\mathcal{G}_{M} \wedge S U(3)\right) \otimes U(1)$.

Let us remark that, for a generic spin-independent central interaction, the "internal" dynamical symmetry group is reduced from $S U(3) \otimes U(1)$ to $S U(2) \otimes U(1)$, spanned the internal energy $\overline{\mathcal{E}}=\mathcal{E} / \mu$ and the internal angular momentum $S_{a}$. Only in special cases the symmetry group is larger than $S U(2) \otimes U(1)$. As is well known, for instance, in the case of Kepler problem, $S U(2) \otimes U(1)$ is enlarged to $S O(4) \otimes U(1)$ for the closed orbits (attractive force, $\mathcal{E}<0$ ), to $E(3) \otimes U(1)(E(3)$ Euclidean group) for parabolic motions (with $\mathcal{E}=0$ ), and to $S O(3,1) \otimes U(1)$ for hyperbolic motion $(\mathcal{E}>0$ ). In the Kepler case, the generators are the internal energy $\overline{\mathcal{E}}$, the spin $S_{a}$ and either $\vec{K}=\vec{R} / \sqrt{2|\mathcal{E}|}(\mathcal{E} \neq 0)$ or $\vec{K}^{\prime}=\vec{R}(\mathcal{E}=0)$, where $\vec{R}=\vec{\pi} \wedge \vec{S} / \mu-G \mu M \vec{r} / r$ is the RungeLenz vector constant of the motion ( $\mu$ reduced mass, $M$ total mass, $G$ graviational constant). Note that for two free particles, the "internal" dynamical symmetry group becomes $U(1) \otimes E(3)$ and the Euclidean transformations are generated by the relative momentum $\vec{\pi}$.

\section{Gauging the $S U(3) \otimes U(1)$ dynamical algebra in the one-time theory}

We have now to implement the idea of describing the extension of the dynamical system by means of both a point-like structure identified with the center-of-mass and an internal structure summarized by its dynamical symmetry.

We shall proceed as follows. First of all, we observe that Newton's mechanics of a point particle may be described using a trivial configuration bundle defined by the absolute-time axis, as base manifold, and the configuration space $R^{3}$, as fiber. The corresponding geometrical structure for a system of two particles having a natural center of mass $(\boldsymbol{x})$ and relative variables $(\boldsymbol{r})$, can still be characterized by a trivial 
fiber bundle defined by the absolute-time axis as base manifold. Yet, the fiber can be expediently viewed as a fiber bundle $(Q)$ by itself, having its base defined by a vector space $R^{3}\left(Q_{x}\right)$, associated to $\boldsymbol{x}$, and a fiber associated to the relative or internal variable $\boldsymbol{r}\left(Q_{r}\right)$. Introducing the dynamics requires that the configuration space $Q$ with coordinates $\{\boldsymbol{x}, \boldsymbol{r}\}$ be replaced by its tangent bundle $T Q$ with coordinates $\{\boldsymbol{x}, \boldsymbol{r} ; \dot{\boldsymbol{x}}, \dot{\boldsymbol{r}}\}$. In this way we get a further trivial bundle over the absolute-time axis. Therefore, since the dynamical algebra acts only on the $\mathcal{L}_{r}(t)$ part of the Lagrangian (see eq (2.3p) with $\mathcal{L}_{r}: T Q_{r} \mapsto R$, in carrying out the gauging of the internal dynamical group one is naturally led to replace the arbitrary constants $\theta^{a}, \xi^{a b}$ of the Noether transformations (2.21) with arbitrary functions $\theta^{a}(\boldsymbol{x}, t), \xi^{a b}(\boldsymbol{x}, t)$ of the center-of-mass coordinates and the absolute-time.

In these conditions, one should expect that, in order the Lagrangian to remain quasi-invariant under the localized $S U(3) \otimes U(1)$ transformations as in eq. (2.22), compensating $S U(3) \otimes U(1)$ Yang-Mills fields, depending on the center-of-mass coordinates $\boldsymbol{x}$, have to be introduced.

We will work out this program by a step-wise procedure. First of all, let us observe that, under the internal transformations

$$
\left\{\begin{array}{l}
\delta_{0}^{g} x^{i}=0 \\
\delta_{0}^{g} r^{a}=\theta^{b}(\boldsymbol{x}) c_{b c}{ }^{a} r^{c}+\sqrt{\frac{\mu}{k}} \xi^{a b}(\boldsymbol{x}) \delta_{b c} \dot{r}^{c},
\end{array}\right.
$$

it follows

$$
\begin{aligned}
\delta_{0}^{g} \mathcal{L}= & \frac{d}{d t}\left[\sqrt{\frac{\mu}{k}} \xi^{a b}(x) \delta_{a c} \delta_{b d}\left(\frac{\mu}{2} \dot{r}^{c} \dot{r}^{d}-\frac{k}{2} r^{c} r^{d}\right)\right] \\
& +\dot{x_{k}} \frac{\partial \theta^{a}}{\partial x^{k}} S_{a}(\boldsymbol{r}, \dot{\boldsymbol{r}})+\dot{x_{k}} \frac{\partial \xi^{c d}}{\partial x^{k}} N_{c d}(\boldsymbol{r}, \dot{\boldsymbol{r}}),
\end{aligned}
$$

where the function $S_{a}(\boldsymbol{r}, \dot{\boldsymbol{r}}), N_{a b}(\boldsymbol{r}, \dot{\boldsymbol{r}})$ are defined in eqs. (2.17) and (2.18). The difference between the present case and the "colored" pseudo-classical particle case is that in the former the Lie algebra generators are realized dynamically in terms of relative variables of the bi-local system, while in the latter they are realized in term of Grassmann variables describing the color charge degrees of freedom of the point like-system in a "quasi-classical" approximation of the standard first-quantized case [3].

Then, since eqs. (3.1) imply:

$$
\begin{aligned}
\delta_{0}^{g} \dot{r}^{a}= & \theta^{b}(\boldsymbol{x}) c_{b c}{ }^{a} \dot{r}^{c}+\sqrt{\frac{\mu}{k}} \xi^{a b}(\boldsymbol{x}) \delta_{b c} \ddot{r}^{c} \\
& +\dot{x}^{k} \frac{\partial \theta^{b}}{\partial x^{k}} c_{b c}{ }^{a} r^{c}+\sqrt{\frac{\mu}{k}} \dot{x}^{k} \frac{\partial \xi^{a b}}{\partial x^{k}} \delta_{b c} \dot{r}^{c},
\end{aligned}
$$


eqs. 2.23) are now replaced by

$$
\left\{\begin{aligned}
\delta_{0}^{g} S_{a}= & c_{a b}{ }^{c} \theta^{b} S_{c}+\xi^{c d} c_{a c}{ }^{b} N_{b d}+\xi^{c d} c_{a d}{ }^{b} N_{c b} \\
& +\sqrt{\frac{\mu}{k}} c_{a b}{ }^{c} \xi^{b d} \delta_{c e} \delta_{d f} r^{e}\left[\frac{\delta \mathcal{L}}{\delta r^{f}}-\frac{d}{d t} \frac{\delta \mathcal{L}}{\delta \dot{r}^{f}}\right] \\
& +\mu \dot{x}^{k} \theta_{, k}^{b} \delta_{c d} c_{a e}{ }^{c} c_{b f}{ }^{d} r^{e} r^{f}+\frac{\mu^{2}}{\sqrt{\mu k}} \dot{x}^{k} \xi_{, k}^{c d} \delta_{c e} \delta_{d f} c_{a b}{ }^{e} r^{b} \dot{r}^{f} \\
\delta_{0}^{g} N_{a b}= & c_{a c}{ }^{d} \theta^{c} \bar{N}_{d b}+c_{b c}{ }^{d} \theta^{c} \bar{N}_{a d}+\frac{1}{2}\left(c_{a c}{ }^{e} \delta_{b d} \xi^{c d}+c_{b c}{ }^{e} \delta_{a d} \xi^{c d}\right) \bar{S}_{e} \\
& -\frac{\mu}{2 k} \delta_{a c} \delta_{b d} \delta_{e f}\left(\dot{r}^{c} \xi^{d e}+\dot{r}^{d} \xi^{c e}\right)\left[\frac{\delta \mathcal{L}}{\delta r^{f}}-\frac{d}{d t} \frac{\delta \mathcal{L}}{\delta \dot{r}^{f}}\right] \\
& +\frac{\mu}{\sqrt{k}} \dot{x}^{k} \theta_{, k}^{c} \delta_{a f} \delta_{b g} c_{c e}{ }^{f} r^{e} \dot{r}^{g}+\frac{\mu^{2}}{k} \dot{x}^{k} \xi_{, k}^{c d} \delta_{a c} \delta_{b e} \delta_{d f} \dot{r}^{e} \dot{r}^{f}
\end{aligned}\right.
$$

where essentially new terms appear which contain the space derivatives of the group parameters. Let us remark that, were it not for the presence of these derivative terms, the introduction of Yang-Mills compensating fields in the simple form:

$$
\mathcal{L} \rightarrow \mathcal{L}^{\prime} \equiv \mathcal{L}+\dot{x}^{k} B_{k}^{(a)}(\boldsymbol{x}) S_{a}(\boldsymbol{r}, \dot{\boldsymbol{r}})+\dot{x}^{k} B_{k}^{(a b)}(\boldsymbol{x}) N_{a b}(\boldsymbol{r}, \dot{\boldsymbol{r}}),
$$

would be already effective to obtain the desired result. In fact, provided that the standard transformation properties be assumed for the Yang-Mills fields $B_{k}^{(a)}$ and $B_{k}^{(a b)}$,

$$
\delta B_{k}^{(a)}(\boldsymbol{x})=\begin{aligned}
(a b) & c_{b c}{ }^{a} \theta^{b}(\boldsymbol{x}) B_{k}^{(c)}(\boldsymbol{x})+\xi^{c d}(\boldsymbol{x})\left[c_{c e}{ }^{a} \delta_{d f} B_{k}^{(e f)}(\boldsymbol{x})+c_{d e}{ }^{a} \delta_{c f} B_{k}^{(e f)}(\boldsymbol{x})\right] \\
& -\frac{\partial \theta^{a}(\boldsymbol{x})}{\partial x^{k}} \\
= & \theta^{c}(\boldsymbol{x})\left[c_{c d}{ }^{a} B_{k}^{(d b)}(\boldsymbol{x})+c_{c d}{ }^{b} B_{k}^{(a d)}(\boldsymbol{x})\right] \\
& +\frac{1}{4} \xi^{c d}(\boldsymbol{x})\left[c_{e c}{ }^{a} \delta_{d}^{b}+c_{e c}{ }^{b} \delta_{d}^{a}+c_{e d}{ }^{a} \delta_{c}^{b}+c_{e d}{ }^{b} \delta_{c}^{a}\right] B_{k}^{(e)}(\boldsymbol{x}) \\
& -\frac{\partial \xi^{a b}(\boldsymbol{x})}{\partial x^{k}}
\end{aligned}
$$

we would have,

$$
\begin{aligned}
\delta_{0}^{g} \mathcal{L}^{\prime}=\frac{d}{d t} & {\left[\sqrt{\frac{\mu}{k}} \xi^{a b} \delta_{a c} \delta_{b d}\left(\frac{\mu}{2} \dot{r}^{c} \dot{r}^{d}+\frac{k}{2 \mu} r^{c} r^{d}\right)\right] } \\
+\dot{x}^{k} & {\left[\sqrt{\frac{\mu}{k}} B_{k}^{(a)} c_{a b} \xi^{b d} \delta_{c e} \delta_{d f} r^{e}-\frac{\mu}{2 k} B_{k}^{(a b)} \delta_{a c} \delta_{b d} \delta_{e f}\left(\dot{r}^{c} \xi^{d e}+\dot{r}^{d} \xi^{c e}\right)\right] . } \\
\cdot & {\left[\frac{\delta \mathcal{L}}{\delta r^{f}}-\frac{d}{d t} \frac{\delta \mathcal{L}}{\delta \dot{r}^{f}}\right] }
\end{aligned}
$$

i.e. a quasi-invariance modulo the equations of motion $\frac{\delta \mathcal{L}}{\delta r^{f}}-\frac{d}{d t} \frac{\delta \mathcal{L}}{\delta \dot{r}^{f}} \stackrel{\circ}{=} 0$ for the quadrupole part.

Therefore, if the Yang-Mills transformations (3.6) have to be preserved, extra terms have to be added to the Lagrangian in order to compensate the extra terms containing $\partial \theta^{a}(\boldsymbol{x}) / \partial x^{k}, \partial \xi^{a b}(\boldsymbol{x}) / \partial x^{k}$ in eqs.(3.4) which arise from the dynamical character of the $S U(3) \otimes U(1)$ realization.

In order to identify these extra terms, let us confine, as a first step, to the subgroup $\mathrm{SU}(2)$ only $\left(\xi^{a b}=0\right)$. In this case, it is evident that the extra terms must have the 
effect of replacing the time derivatives of the relative variables $\left(\dot{r}^{a}\right)$ with the covariant derivative

$$
D r^{a}=\dot{r}^{a}+\dot{x}^{k} B_{k}^{(b)}(\boldsymbol{x}) c_{b c}{ }^{a} r^{c} .
$$

Then, the quasi-invariant Lagrangian would result

$$
\mathcal{L}_{S U(2)}=\frac{M}{2} \delta_{i j} \dot{x}^{i} \dot{x}^{j}-\frac{k}{2 \mu} \delta_{a b} r^{a} r^{b}+\frac{\mu}{2} \delta_{a b} D r^{a} D r^{b} .
$$

Since the momenta are

$$
\left\{\begin{aligned}
p_{i} & =M \delta_{i j} \dot{x}^{j}+\mu B_{i}^{(b)}(\boldsymbol{x}) c_{b c}{ }^{a} r^{c} \delta_{a d} D r^{d} \\
\pi_{a} & =\mu \delta_{a b} D r^{b}
\end{aligned}\right.
$$

we would get a minimal coupling in the Hamiltonian:

$$
\begin{aligned}
\bar{H}_{S U(2)}= & \frac{1}{2 M} \delta^{i j}\left[P_{i}-B_{i}^{(a)}(\boldsymbol{x}) S_{a}(\boldsymbol{r}, \boldsymbol{\pi})\right]\left[P_{j}-B_{j}^{(a)}(\boldsymbol{x}) S_{a}(\boldsymbol{r}, \boldsymbol{\pi})\right] \\
& +\frac{1}{2 \mu} \delta^{a b} \pi_{a} \pi_{b}+\frac{k}{2 \mu} \delta_{a b} r^{a} r^{b} .
\end{aligned}
$$

Note that $\bar{H}_{S U(2)}$ is indeed invariant under the phase-space transformations generated by $\theta^{a}(\boldsymbol{x}) \bar{S}_{a}$ if $\delta B_{k}^{(a)}(\boldsymbol{x})$ is given by eqs. 3.6) with $\xi^{a b}(\boldsymbol{x})=0$.

It is now straightforward to check, via eqs. (3.6), that the generalized minimally coupled Hamiltonian

$$
\begin{aligned}
& \bar{H}_{S U(3) \otimes U(1)}= \frac{1}{2 M} \delta^{i j}\left[P_{i}-B_{i}^{(a)}(\boldsymbol{x}) S_{a}-B_{i}^{(a b)}(\boldsymbol{x}) N_{a b}\right] \\
& {\left[P_{i}-B_{j}^{(a)}(\boldsymbol{x}) S_{a}-B_{j}^{(a b)}(\boldsymbol{x}) N_{a b}\right] } \\
&+\frac{1}{2 \mu} \delta^{a b} \pi_{a} \pi_{b}+\frac{k}{2} \delta_{a b} r^{a} r^{b}
\end{aligned}
$$

is invariant under the complete $S U(3) \otimes U(1)$ transformations generated by $\theta^{a}(\boldsymbol{x}) \bar{S}_{a}+$ $\xi^{a b}(\boldsymbol{x}) \bar{N}_{a b}$. Actually, we have

$$
\bar{\delta}_{0} P_{i}=-\frac{\partial \theta^{a}(\boldsymbol{x})}{\partial x^{i}} \bar{S}_{a}-\frac{\partial \xi^{a b}(\boldsymbol{x})}{\partial x^{i}} \bar{N}_{a b}
$$

so that $\bar{\delta}_{0}\left[P_{k}-B_{k}^{(a)}(\boldsymbol{x}) S_{a}-B_{k}^{(a b)}(\boldsymbol{x}) N_{a b}\right]=0$. Then, since

$$
\bar{\delta}_{0}\left[\frac{1}{2 \mu} \delta^{a b} \pi_{a} \pi_{b}+\frac{k}{2} \delta_{a b} r^{a} r^{b}\right]=0,
$$

$\bar{H}_{S U(3) \otimes U(1)}$ is seen to be strictly invariant.

To obtain the quasi-invariant Lagrangian $\mathcal{L}_{S U(3) \otimes U(1)}$, we have simply to perform the inverse Legendre transformation defined via the first half of Hamilton's equations

$$
\left\{\begin{aligned}
\dot{x}^{i} \stackrel{\circ}{=} \frac{\partial \bar{H}}{\partial P_{i}} & =\frac{1}{M} \delta^{i j}\left[P_{j}-B_{j}^{(a)}(\boldsymbol{x}) \bar{S}_{a}-B_{j}^{(a b)}(\boldsymbol{x}) \bar{N}_{a b}\right] \\
\dot{r}^{a} \stackrel{\circ}{=} \frac{\partial \bar{H}}{\partial \pi_{a}} & =\delta^{a b} \frac{\pi_{b}}{\mu}-\frac{\partial \bar{H}}{\partial P_{k}}\left[B_{k}^{(c)}(\boldsymbol{x}) \frac{\partial \bar{S}_{c}}{\partial \pi_{a}}+B_{k}^{(c d)}(\boldsymbol{x}) \frac{\partial \bar{N}_{c d}}{\partial \pi_{a}}\right] \\
& =\delta^{a b} \frac{\pi_{b}}{\mu}-\dot{x}^{k}\left[B_{k}^{(c)}(\boldsymbol{x}) \frac{\partial \bar{S}_{c}}{\partial \pi_{a}}+B_{k}^{(c d)}(\boldsymbol{x}) \frac{\partial \bar{N}_{c d}}{\partial \pi_{a}}\right]
\end{aligned}\right.
$$


(where the symbol $\stackrel{\circ}{=}$ means "evaluated on the solutions of the equations of motion").

Since, in phase space, the generators $\bar{S}_{a}, \bar{N}_{a b}$ have still the form they have in eqs. 2.17), (2.18), because of the second of eqs(3.14), we can write

$$
\Xi^{a b}(\boldsymbol{x}) \pi_{b}=\mu D r^{a}
$$

where

$$
\Xi^{a b}(\boldsymbol{x})=\delta^{a b}-\sqrt{\frac{\mu}{k}} \dot{x}^{k} B_{k}^{(a b)}(\boldsymbol{x})
$$

Therefore, we obtain

$$
\pi_{a}=\mu\left|\Xi^{-1}\right|_{a b}(\boldsymbol{x}) D r^{b} .
$$

Then, the first of eqs. (3.14) gives

$$
P_{i}=M \delta_{i j} \dot{x}^{j}+B_{i}^{(a)}(\boldsymbol{x}) \bar{S}_{a}+B_{i}^{(a b)}(\boldsymbol{x}) \bar{N}_{a b},
$$

and we obtain the Lagrangian as:

$$
\begin{aligned}
\mathcal{L}_{S U(3) \otimes U(1)} & =P_{k} \dot{x}^{k}+\pi_{a} \dot{r}^{a}-\bar{H}_{S U(3) \otimes U(1)} \\
& =\frac{M}{2} \delta_{i j} \dot{x}^{i} \dot{x}^{j}+\frac{\mu}{2}\left|\Xi^{-1}\right|_{a b} D r^{a} D r^{b}-\frac{k}{2} \Xi^{a b} \delta_{a c} \delta_{b d} r^{c} r^{d} .
\end{aligned}
$$

Finally, using eq.(2.18), we get

$$
B_{k}^{(a b)}(\boldsymbol{x}) \bar{N}_{a b}=B_{k}^{(a b)}(\boldsymbol{x}) \overline{\mathcal{Q}}_{a b}+B_{k}^{\prime}(\boldsymbol{x}) \frac{\mathcal{E}^{\prime}}{3}
$$

and

$$
\left\{\begin{aligned}
B_{k}^{(a b)}(\boldsymbol{x}) & =B_{k}^{(a b)}(\boldsymbol{x})-\frac{1}{3} \delta^{a b} \delta_{c d} B_{k}^{(c d)}(\boldsymbol{x}) \\
B_{k}^{\prime}(\boldsymbol{x}) & =\delta_{c d} B_{k}^{(c d)}(\boldsymbol{x}) .
\end{aligned}\right.
$$

Eqs.(3.21) identify the Yang-Mills fields, associated to the quadrupole moment and to the internal energy, respectively, which contribute to the potential energy term through the symmetric tensor $\Xi^{a b}(\boldsymbol{x})$. Its inverse $\left|\Xi^{-1}\right|_{a b}$ behaves as a dynamically induced "internal metric" for the scalar product of the contravariant vector $D \boldsymbol{r}=$ $\left(D r^{a}\right)$. On the other hand, the $\mathrm{SU}(2)$ Yang-Mills fields determine the "internal spin connection" $\left({ }^{S p i n} \Gamma_{k a}^{b}=B_{k}^{(c)} c_{c a}^{b}\right)$ and its associated "internal covariant derivative" $D r^{a}$.

The $S U(3) \otimes U(1)$ generators, expressed in configuration space, become:

$$
\left\{\begin{aligned}
\mathcal{E}^{\prime}= & \frac{1}{2}\left(\left.\left.\frac{\mu^{2}}{\sqrt{\mu k}} \delta^{a b}\right|_{\Xi^{-1}}\right|_{a c}(\boldsymbol{x})\left|\Xi^{-1}\right|_{b d}(\boldsymbol{x}) D r^{c} D r^{d}+\sqrt{\mu k} \delta_{a b} r^{a} r^{b}\right) \\
S_{a}= & \mu c_{a b}{ }^{b} r^{b}\left|\Xi^{-1}\right|_{c d}(\boldsymbol{x}) D r^{d} \\
\mathcal{Q}_{a b}= & {\left[\frac{\mu^{2}}{2 \sqrt{\mu k}}\left|\Xi^{-1}\right|_{a c}(\boldsymbol{x}) D r^{c}\left|\Xi^{-1}\right|_{b d}(\boldsymbol{x}) D r^{d}+\frac{\sqrt{\mu k}}{2} \delta_{a c} \delta_{b d} r^{c} r^{d}\right] } \\
& -\delta_{a b} \frac{\mathcal{E}^{\prime}}{3} .
\end{aligned}\right.
$$

Now, under the $S U(3) \otimes U(1)$ transformations defined by (3.6) and:

$$
\delta r^{a}=c_{b c}{ }^{c} \theta^{b}(\boldsymbol{x}) r^{c}+\frac{\mu}{\sqrt{k}} \xi^{a b}(\boldsymbol{x})\left|\Xi^{-1}\right|_{b c}(\boldsymbol{x}) D r^{c}
$$


we get

$$
\begin{aligned}
\delta \mathcal{L}_{S U(3) \otimes U(1)} & = \\
& =\frac{d}{d t}\left[\sqrt{\frac{\mu}{k}} \xi^{a b}(\boldsymbol{x})\left(\frac{\mu}{2}\left|\Xi^{-1}\right|_{a c}(\boldsymbol{x}) D r^{c}\left|\Xi^{-1}\right|_{b d}(\boldsymbol{x}) D r^{d}-\frac{k}{2} \delta_{a c} \delta_{b d} r^{c} r^{d}\right)\right] .
\end{aligned}
$$

We want to impose now invariance (quasi-invariance) of the theory with respect to local gauge variations dependent on time, besides the center-of-mass coordinates, i.e. for transformations of the form:

$$
\delta r^{a}=c_{b c}^{a} \theta^{b}(\boldsymbol{x}, t) r^{c}+\sqrt{\frac{\mu}{k}} \xi^{a b}(\boldsymbol{x}, t)\left|\Xi^{-1}\right|_{b c}(\boldsymbol{x}, t) D r^{c} .
$$

This task cannot be dealt with in the non reparametrization-invariant Hamiltonian picture. It is easy to verify, however, that the only needed modification is the introduction of additional 0-components for the $S U(3) \otimes U(1)$ fields $B_{i}^{(a)}$ and $B_{i}^{(a b)}$, along with a modification of the transformation properties (3.6), in the form

$$
\left\{\begin{aligned}
\delta B_{k}^{(a)}(\boldsymbol{x}, t)= & -\frac{\partial \theta^{a}(\boldsymbol{x}, t)}{\partial x^{k}}+c_{b c}{ }^{a} \theta^{b}(\boldsymbol{x}, t) B_{k}^{(c)}(\boldsymbol{x}, t) \\
& +\xi^{c d}(\boldsymbol{x}, t)\left[c_{c e}{ }^{a} \delta_{d f} B_{k}^{(e f)}(\boldsymbol{x}, t)+c_{d e}{ }^{a} \delta_{c f} B_{k}^{(e f)}(\boldsymbol{x}, t)\right] \\
\delta B_{0}^{(a)}(\boldsymbol{x}, t)= & -\frac{\partial \theta^{a}(\boldsymbol{x}, t)}{\partial t}+c_{b c}{ }^{a} \theta^{b}(\boldsymbol{x}, t) B_{0}^{(c)}(\boldsymbol{x}, t) \\
& +\xi^{c d}(\boldsymbol{x}, t)\left[c_{c e}{ }^{a} \delta_{d f} B_{0}^{(e f)}(\boldsymbol{x}, t)+c_{d e}{ }^{a} \delta_{c f} B_{0}^{(e f)}(\boldsymbol{x}, t)\right] \\
\delta B_{k}^{(a b)}(\boldsymbol{x}, t)= & \theta^{c}(\boldsymbol{x}, t)\left[c_{c d}{ }^{a} B_{k}^{(d b)}(\boldsymbol{x}, t)+c_{c d}{ }^{b} B_{k}^{(a d)}(\boldsymbol{x}, t)\right] \\
& +\frac{1}{4} \xi^{c d}(\boldsymbol{x}, t)\left[c_{e c}{ }^{a} \delta_{d}^{b}+c_{e c}{ }^{b} \delta_{d}^{a}+c_{e d}{ }^{a} \delta_{c}^{b}+c_{e d}{ }^{b} \delta_{c}^{a}\right] B_{k}^{(e)}(\boldsymbol{x}, t) \\
& -\frac{\partial \xi^{a b}(\boldsymbol{x}, t)}{\partial x^{k}} \\
\delta B_{0}^{(a b)}(\boldsymbol{x}, t)= & \theta^{c}(\boldsymbol{x}, t)\left[c_{c d}{ }^{a} B_{0}^{(d b)}(\boldsymbol{x}, t)+c_{c d}{ }^{b} B_{0}^{(a d)}(\boldsymbol{x}, t)\right] \\
& +\frac{1}{4} \xi^{c d}(\boldsymbol{x}, t)\left[c_{e c}{ }^{a} \delta_{d}^{b}+c_{e c}{ }^{b} \delta_{d}^{a}+c_{e d}{ }^{a} \delta_{c}^{b}+c_{e d}{ }^{b} \delta_{c}^{a}\right] B_{0}^{(e)}(\boldsymbol{x}, t) \\
& -\frac{\partial \xi^{a b}(\boldsymbol{x}, t)}{\partial t},
\end{aligned}\right.
$$

and a redefinition of $D r^{a}$ and $\Xi^{a b}(\boldsymbol{x})$ to take into account the additional time components:

$$
\begin{aligned}
D r^{a} & =\dot{r}^{a}+\dot{x}^{k} B_{k}^{(b)}(\boldsymbol{x}, t) c_{b c}{ }^{a} r^{c}+B_{0}^{(b)}(\boldsymbol{x}, t) c_{b c}{ }^{a} r^{c} \\
\Xi^{a b}(\boldsymbol{x}, t) & =\delta^{a b}-\sqrt{\frac{\mu}{k}}\left[\dot{x}^{k} B_{k}^{(a b)}(\boldsymbol{x}, t)-B_{0}^{(a b)}(\boldsymbol{x}, t)\right] .
\end{aligned}
$$

Then, we obtain the Lagrangian:

$\mathcal{L}_{S U(3) \otimes U(1)}=\frac{M}{2} \delta_{i j} \dot{x}^{i} \dot{x}^{j}+\frac{\mu}{2}\left|\Xi^{-1}\right|_{a b}(\boldsymbol{x}, t) D r^{a} D r^{b}-\frac{k}{2} \Xi^{a b}(\boldsymbol{x}, t) \delta_{a c} \delta_{b d} r^{c} r^{d}$,

which is quasi-invariant under the transformation (3.25). In fact, we have:

$$
\begin{aligned}
& \delta \mathcal{L}_{S U(3) \otimes U(1)}= \\
& \quad=\frac{d}{d t}\left[\sqrt{\frac{\mu}{k}} \xi^{a b}(\boldsymbol{x}, t)\left(\frac{\mu}{2}\left|\Xi^{-1}\right|_{a c}(\boldsymbol{x}, t) D r^{c}\left|\Xi^{-1}\right|_{b d}(\boldsymbol{x}, t) D r^{d}-\frac{k}{2} \delta_{a c} \delta_{b d} r^{c} r^{d}\right)\right] .
\end{aligned}
$$


It is worth stressing the peculiar role played by the "internal" covariant derivative and the "internal" metric in the Lagrangian expression (3.28).

Within the Hamiltonian picture, the generalization above corresponds to the substitution rule

$$
P_{i} \mapsto \mathcal{P}_{i}=P_{i}-B_{i}^{(a)}(\boldsymbol{x}, t) S_{a}-B_{i}^{(a b)}(\boldsymbol{x}, t) N_{a b},
$$

and, consequently, to

$$
H \mapsto \mathcal{H}=H[\mathcal{P}]-B_{0}^{(a)}(\boldsymbol{x}, t) S_{a}-B_{0}^{(a b)}(\boldsymbol{x}, t) N_{a b} .
$$

Let us remark that the trace parts $\left(B_{k}^{\prime}(\boldsymbol{x}, t), B_{0}^{\prime}(\boldsymbol{x}, t)\right)$ of the $B$-fields transform separately as

$$
\begin{aligned}
\delta B_{k}^{\prime}(\boldsymbol{x}, t) & =-\frac{\partial}{\partial x^{k}}\left[\delta_{a b} \xi^{a b}(\boldsymbol{x}, t)\right] \\
\delta B_{0}^{\prime}(\boldsymbol{x}, t) & =-\frac{\partial}{\partial t}\left[\delta_{a b} \xi^{a b}(\boldsymbol{x}, t)\right],
\end{aligned}
$$

as indeed it must be, due to the Abelian character of the U(1) subgroup generated by the internal energy $\mathcal{E}^{\prime}$, to which these fields are associated.

Following a similar way of deduction, is it possible to find out the time variation law for the conserved charge. Let $\left(W_{i}\right)_{u}$ and $q_{i}$ be the gauge-fields and the position variables, respectively, and let us denote their variation under the $\delta$ transformations by

$$
\begin{array}{ll}
\delta q_{i} & =\epsilon \phi_{i}[q, \dot{q}] \\
\delta\left(W_{i}\right)_{u} & =\epsilon \Phi_{i, u}\left[\left(W_{i}\right)_{u}\right] .
\end{array}
$$

Moreover, let us impose that the variation of the Action under the transformation of these quantities be a total time derivativet:

$$
\delta S\left[q, \dot{q},\left(W_{i}\right)_{u}\right]=\int d t \delta \mathcal{L}=\int d t \frac{d}{d t}[\epsilon F] .
$$

Finally, let us rewrite this variation of the Lagrangian in the form:

$\delta S\left[q, \dot{q},\left(W_{i}\right)_{u}\right]=\int d t\left\{\left[\frac{\partial \mathcal{L}}{\partial q_{i}}-\frac{d}{d t} \frac{\partial \mathcal{L}}{\partial \dot{q}_{i}}\right] \epsilon \phi_{i}+\frac{d}{d t}\left[\frac{\partial \mathcal{L}}{\partial \dot{q}_{i}} \epsilon \phi_{i}\right]+\frac{\partial \mathcal{L}}{\partial\left(W_{i}\right)_{u}} \epsilon \Phi_{i, u}\right\}$.

Then, since the expressions (3.33) and (3.34) must be equal, putting $\epsilon$ equal to constant and taking into account the Euler-Lagrange equations $\frac{\partial \mathcal{L}}{\partial q_{i}}-\frac{d}{d t} \frac{\partial \mathcal{L}}{\partial \dot{q}_{i}}=0$, it follows

$$
\frac{d}{d t}\left[F-\frac{\partial \mathcal{L}}{\partial \dot{q}_{i}} \phi_{i}\right]=\frac{\partial \mathcal{L}}{\partial\left(W_{i}\right)_{u}} \Phi_{i, u} .
$$

Note, in particular, that, in the case of the oscillator, the time variations of the standard generators of $S U(3) \otimes U(1)$, corresponding to this way of describing the transformations, are

$$
\begin{aligned}
\frac{d}{d t} S_{a}= & c_{a b}{ }^{c} S_{c}\left(\dot{x}^{i} B_{i}^{(b)}+B_{0}^{(b)}\right)+2 c_{a c}{ }^{b} N_{b d}\left(\dot{x}^{i} B_{i}^{(c d)}+B_{0}^{(c d)}\right) \\
\frac{d}{d t} N_{a b}= & \left(c_{a c}{ }^{d} \bar{N}_{d b}+c_{b c}{ }^{d} \bar{N}_{a d}\right)\left(\dot{x}^{i} B_{i}^{(c)}+B_{0}^{(c)}\right) \\
& +\frac{1}{2}\left(c_{a c}{ }^{e} \delta_{b d}+c_{b c}^{e} \delta_{a d}\right) \bar{S}_{e}\left(\dot{x}^{i} B_{i}^{(c d)}+B_{0}^{(c d)}\right) .
\end{aligned}
$$

$\dagger$ As is well known, from this hypothesis, it follows that $\delta$ is a symmetry of the system 


\section{Gauging the extended Galilei Group for the free particle}

In reference [1] we have shown that by gauging à la Utiyama [2] the realization of the extended Galilei group associated to a non-relativistic free particle of mass $M$, one is led to the Lagrangian:

$$
\mathcal{L}_{M}^{g}(t)=\frac{1}{\Theta} \frac{M}{2}\left[g_{i j} \dot{x}^{i} \dot{x}^{j}+2 A_{i} \dot{x}^{i}+2 A_{0}\right] .
$$

This Lagrangian is quasi-invariant under the mass-point coordinate transformations

$$
\left\{\begin{aligned}
\delta_{0} t= & 0 \\
\delta_{0} x^{i}= & \varepsilon(t) \dot{x}^{i}+\eta^{i}(\boldsymbol{x}, t)-t v^{i}(\boldsymbol{x}, t) \\
\delta_{0} \dot{x}^{i}= & \frac{d}{d t}\left[\varepsilon(t) \dot{x}^{i}\right]+\dot{x}^{k} \frac{\partial}{\partial x^{k}}\left[\eta^{i}(\boldsymbol{x}, t)-t v^{i}(\boldsymbol{x}, t)\right] \\
& +\frac{\partial}{\partial t}\left[\eta^{i}(\boldsymbol{x}, t)-t v^{i}(\boldsymbol{x}, t)\right]
\end{aligned}\right.
$$

and the field transformations

$$
\left\{\begin{aligned}
\delta_{0} \Theta & =\hat{\delta}_{0} \Theta=\dot{\varepsilon} \Theta \\
\delta_{0} A_{0} & =\hat{\delta}_{0} A_{0}-\varepsilon \frac{\partial A_{0}}{\partial t}+A_{i, j} \tilde{\eta}^{j} \\
& =2 \dot{\varepsilon} A_{0}-A_{i} \frac{\partial \tilde{\eta}^{i}}{\partial t}+\Theta \frac{\partial \mathcal{F}}{\partial t} \\
\delta_{0} A_{i} & =\hat{\delta}_{0} A_{i}-\varepsilon \frac{\partial A_{i}}{\partial t}+A_{i, j} \tilde{\eta}^{j} \\
& =\dot{\varepsilon} A_{i}-A_{j} \frac{\partial \tilde{\eta}^{j}}{\partial x^{i}}-g_{i j} \frac{\partial \tilde{\eta}^{j}}{\partial t}+\Theta \frac{\partial \mathcal{F}}{\partial x^{i}}
\end{aligned}\right.
$$

in the sense that

$$
\delta_{0} \mathcal{L}_{M}^{g}=\frac{d \mathcal{F}}{d t}+\varepsilon \frac{d\left[\mathcal{L}_{M}^{g}\right]}{d t}
$$

holds, so that

$$
\Delta \mathcal{S}_{M}^{g}=\int_{t_{1}}^{t_{2}} d t\left[\frac{d \mathcal{F}}{d t}+\frac{d\left[\varepsilon \mathcal{L}_{M}^{g}\right]}{d t}\right]=0,
$$

where $\mathcal{F}=M g_{i j} v^{i}(x, t) x^{j}$ is the cocycle term connected to the central extension of the group.

For later use it is instrumental to report explicitly the deduction of this result in the reparameterization-invariant Hamiltonian description. Let $\boldsymbol{x}(\tau), t(\tau)$, be the space and time coordinates of the free mass-point. The Lagrangian and the Action can be written

$$
\begin{aligned}
\hat{\mathcal{L}}_{M}(\lambda) & =\frac{1}{2} M \frac{\delta_{i j} x^{i}(\lambda) x^{\prime j}(\lambda)}{t^{\prime}(\lambda)}, \\
\hat{\mathcal{A}}_{M} & =\int_{\lambda_{1}}^{\lambda_{2}} d \lambda \hat{\mathcal{L}}_{M}(\lambda),
\end{aligned}
$$


where $f^{\prime}(\lambda) \equiv \frac{d}{d \lambda} f(\lambda)$. The canonical momenta are

$$
\left\{\begin{array}{l}
\hat{E}=-\frac{\partial \hat{\mathcal{L}}}{\partial t^{\prime}}=\frac{M}{2} \frac{\delta_{i j}{x^{\prime}}^{i}(\lambda) x^{\prime j}(\lambda)}{t^{\prime 2}(\lambda)}=\frac{1}{2 M} \delta^{i j} \hat{p}_{i} \hat{p}_{j} \\
\hat{p}_{i}=\frac{\partial \hat{\mathcal{L}}}{\partial{x^{\prime}}^{i}}=M \frac{\delta_{i j} x^{\prime j}(\lambda)}{t^{\prime}(\lambda)}=M \delta_{i j} \dot{x}^{j}(t)=p_{i}
\end{array}\right.
$$

where we have defined the Poisson brackets so that:

$$
\begin{aligned}
\{t(\lambda), \hat{E}(\lambda)\}^{\prime} & =-1 \\
\left\{x^{i}(\lambda), \hat{p}_{j}(\lambda)\right\}^{\prime} & =\delta_{j}^{i} .
\end{aligned}
$$

In the enlarged phase-space, coordinatized by $t, x^{i}, \hat{E}, \hat{p}_{i}$, we get a vanishing canonical Hamiltonian and the first-class constraint

$$
\hat{\chi} \equiv \hat{E}-\frac{1}{2 M} \delta^{i j} \hat{p}_{i} \hat{p}_{j} \approx 0
$$

The constraint $\hat{\chi}$ generates the following transformations of the configurational variables: $\delta t(\lambda)=-\alpha(\lambda), \quad \delta x^{i}(\lambda)=-\alpha(\lambda) \frac{1}{M} \delta^{i j} \hat{p}_{j}$. This corresponds to the reparameterization gauge transformation $\lambda \rightarrow \lambda+\alpha(\lambda) / t^{\prime}$. The Lagrangian is obviously quasi-invariant under this operation since

$$
\delta \hat{\mathcal{L}}_{M}=\frac{d}{d \lambda}\left[\frac{-\alpha(\lambda)}{t^{\prime}} \hat{\mathcal{L}}_{M}\right]
$$

The canonical generators of the extended Galilei group are now:

$\hat{\mathcal{H}}=\hat{E}, \quad \hat{\mathcal{P}}_{i}=\hat{p}_{i}, \quad \hat{\mathcal{K}}_{i}=M \delta_{i j} x^{j}-t \hat{p}_{i}, \quad \hat{\mathcal{J}}_{i}=c_{i j}{ }^{k} x^{j} \hat{p}_{k}, \quad \hat{\mathcal{M}}=M$,

and satisfy the Lie-algebra (2.12) with the primed Poisson-brackets (4.8). Consequently, the generator of the complete phase-space Galilei transformation $\hat{\bar{\delta}}$, which is now given by

$$
\begin{aligned}
\hat{\bar{G}} & =\varepsilon \hat{E}+\varepsilon^{i} \hat{p}_{i}+\omega^{i} \hat{\bar{J}}_{i}+v^{i} \hat{\bar{K}}_{i} \\
& =\varepsilon \hat{E}+\left(\eta^{i}-t v^{i}\right) \hat{p}_{i}+M \delta_{i j} v^{i} x^{j}
\end{aligned}
$$

yields the following "equal- $\lambda$ " infinitesimal transformations:

$$
\left\{\begin{aligned}
\hat{\bar{\delta}} \lambda & =0 \\
\hat{\bar{\delta}} t & =\{t, \hat{\bar{G}}\}=-\varepsilon=\hat{\delta}_{0} t=\delta t \\
\hat{\bar{\delta}} x^{i} & =\left\{x^{i}, \overline{\bar{G}}\right\}=\varepsilon^{i}+c_{j k}{ }^{i} \omega^{j} x^{k}-t v^{i}=\hat{\delta}_{0} x^{i}=\delta x^{i} .
\end{aligned}\right.
$$

We have now $\left.\hat{\delta} \hat{p}_{i}\right|_{\hat{p}=\partial \hat{\mathcal{L}}_{M} / \partial x^{\prime}}=\hat{\delta}\left[\frac{M \delta_{i j} x^{\prime j}}{t^{\prime}}\right]$, without any use of Euler-Lagrange equations. On the other hand, under the transformations (4.13), it follows:

$$
\hat{\bar{\delta}} \hat{\mathcal{L}}_{M}=\frac{d}{d \lambda}\left[-M \delta_{i j} v^{i} x^{j}\right]
$$

and

$$
\hat{\bar{\delta}} \hat{\chi}=0
$$


so that the canonical generators (4.11) are constants of the motion, as they must be. Moreover, the (first-class) constraint (4.9) is Galilei invariant, and the quasi-invariance of the Lagrangian is an effect of the central-charge term alone.

We want now to impose the condition that the theory be invariant under the localized Galilei transformation generated by:

$$
\begin{aligned}
\hat{\bar{G}} & =\varepsilon(t) \hat{E}+\varepsilon^{i}(\boldsymbol{x}, t) \hat{p}_{i}+\omega^{i}(\boldsymbol{x}, t) \hat{\bar{J}}_{i}+v^{i}(\boldsymbol{x}, t) \hat{\bar{K}}_{i} \\
& =\varepsilon(t) \hat{E}+\left[\eta^{i}(\boldsymbol{x}, t)-t v^{i}(\boldsymbol{x}, t)\right] \hat{p}_{i}+M \delta_{i j} v^{i}(\boldsymbol{x}, t) x^{j},
\end{aligned}
$$

i.e.:

$$
\left\{\begin{array}{l}
\hat{\bar{\delta}}_{0} t(\lambda)=-\varepsilon(t(\lambda)) \\
\hat{\bar{\delta}}_{0} x^{i}(\lambda)=\eta^{i}(\boldsymbol{x}, t)-t v^{i}(\boldsymbol{x}, t) \\
\hat{\bar{\delta}}_{0} \hat{p}_{i}=-\hat{p}_{k} \frac{\partial}{\partial x^{i}}\left[\eta^{k}(\boldsymbol{x}, t)-t v^{k}(\boldsymbol{x}, t)\right]-M \frac{\partial}{\partial x^{i}}\left[\delta_{l k} x^{l} v^{k}(\boldsymbol{x}, t)\right] \\
\hat{\bar{\delta}}_{0} \hat{E}=\hat{E} \frac{d \varepsilon(t)}{d t}+\hat{p}_{i} \frac{\partial}{\partial t}\left[\eta^{i}(\boldsymbol{x}, t)-t v^{i}(\boldsymbol{x}, t)\right]+M \frac{\partial}{\partial t}\left[\delta_{i j} x^{i} v^{j}(\boldsymbol{x}, t)\right] .
\end{array}\right.
$$

Let us consider first the phase-space approach. By introducing the minimal coupling (see Ref.[1], Section 3):

$$
\left\{\begin{aligned}
P_{i} & \mapsto \mathcal{P}_{a}=\mathbf{H}_{a}^{k}(\boldsymbol{x}, t)\left(P_{k}-\frac{M}{\Theta} A_{k}(\boldsymbol{x}, t)\right) \\
E & \mapsto \mathcal{E}=\frac{1}{\Theta}\left(E-\frac{M}{\Theta} A_{0}(\boldsymbol{x}, t)\right)
\end{aligned}\right.
$$

we get the following gauge constraint:

$$
\hat{\chi}_{g}=\frac{1}{\Theta}\left[\hat{E}+\frac{M}{\Theta} A_{0}\right]-\frac{g^{i j}}{2 m}\left[\hat{p}_{i}-\frac{M}{\Theta} A_{i}\right]\left[\hat{p}_{j}-\frac{M}{\Theta} A_{j}\right] \simeq 0 .
$$

Note that the counterpart of the second equation (4.18) in the standard (non reparameterization-invariant) picture is:

$$
H \mapsto \mathcal{H}=\Theta(t) H[\mathcal{P}]-\frac{M}{\Theta} A_{0}(\boldsymbol{x}, t) .
$$

Since, under the transformation (4.17), we have

$$
\begin{aligned}
\hat{\delta}_{0} \hat{\chi} & =\frac{1}{\Theta} \hat{\bar{\delta}} \hat{E}-\frac{1}{\Theta^{2}}\left[\hat{E}+\frac{M}{\Theta} A_{0}\right] \hat{\delta}_{0} \Theta+\frac{1}{\Theta^{2}} M \hat{\delta}_{0} A_{0} \\
& -\frac{1}{2 M}\left(\hat{\delta}_{0} g^{i j}\right)\left[\hat{p}_{i}-\frac{M}{\Theta} A_{i}\right]\left[\hat{p}_{j}-\frac{M}{\Theta} A_{j}\right] \\
& -\frac{g^{i j}}{M}\left[\hat{p}_{i}-\frac{M}{\Theta} A_{i}\right]\left[\hat{\bar{\delta}} \hat{p}_{j}-\frac{M}{\Theta} \hat{\delta}_{0} A_{j}+\frac{M}{\Theta^{2}} \hat{\delta}_{0} \Theta A_{j}\right],
\end{aligned}
$$

the invariance of the constraint

$$
\hat{\delta}_{0} \hat{\chi}_{g}=0,
$$

is satisfied provided that the fields transform according to

$$
\left\{\begin{aligned}
\hat{\delta}_{0} \Theta & =\dot{\epsilon}(t) \Theta(t) \\
\hat{\delta}_{0} g_{i j} & =-\frac{\partial \tilde{\eta}^{k}(\mathbf{x}, t)}{\partial x^{i}} g_{k j}-\frac{\partial \tilde{\eta}^{k}(\mathbf{x}, t)}{\partial x^{i}} g_{k j} \\
\hat{\delta}_{0} A_{0} & =2 \dot{\varepsilon} A_{0}-A_{i} \frac{\partial \tilde{\eta}^{i}}{\partial t}-\Theta \frac{\partial}{\partial t}\left[g_{i j} v^{i} x^{j}\right] \\
\hat{\delta}_{0} A_{i} & =\dot{\varepsilon} A_{i}-A_{j} \frac{\partial \tilde{\eta}^{j}}{\partial x^{i}}-g_{i j} \frac{\partial \tilde{\eta}^{j}}{\partial t}-\Theta \frac{\partial}{\partial x^{i}}\left[g_{i j} v^{i} x^{j}\right] .
\end{aligned}\right.
$$


The gauge reparameterization-invariant Lagrangian corresponding to the constraints (4.19) is:

$$
\hat{\mathcal{L}}_{M}^{g}(\lambda)=\frac{1}{\Theta t^{\prime}} \frac{M}{2}\left[g_{i j} x^{\prime i} x^{\prime j}+2 A_{i} x^{\prime} t^{\prime}+2 A_{0} t^{\prime} t^{\prime}\right]
$$

with

$$
\hat{\delta} \hat{\mathcal{L}}_{M}^{g}=\frac{d \mathcal{F}}{d \lambda},
$$

and it corresponds indeed to the "standard" Action (4.1). It is worth remarking that, while in the standard Lagrangian picture the specific form of the cocycle term $\mathcal{F}$ must be guessed, the latter is explicitly determined within the Hamiltonian picture. In fact, since the algebraic properties are essential in the Hamiltonian formalism, the cocycle term is a necessary consequence of the central extension of the group.

Note finally that, were it not for the presence of the einbein-like component $\Theta$, the modified Hamiltonian constraint (4.9) could have been made invariant only in the weak sense $\hat{\delta} \hat{\chi}_{g}=\varepsilon \hat{\chi}_{g} \approx 0$.

\section{Gauging the maximal symmetry group in the one-time theory}

We are now ready to gauge the Galilei kinematical plus the internal dynamical group of the harmonic oscillator in a unified way. As shown in Section 1, the algebra of the maximal dynamical symmetry of the harmonic oscillator is the semi-direct sum of the kinematical extended Galilei algebra times the dynamical $S U(3) \otimes U(1)$ algebra given by (2.12,2.19,2.24). We proceed by letting the infinitesimal parameters of the transformations (2.15) and (2.21) to become dependent on the center-of-mass coordinates and time; then, by searching for a new Action invariant (quasi-invariant) under the transformations

$$
\left\{\begin{aligned}
\delta_{0} x^{i} & =\epsilon(t) \dot{x}^{i}+\epsilon^{i}(\boldsymbol{x}, t)+c_{j k}{ }^{l} \omega^{j}(\boldsymbol{x}, t) x^{k}-t v^{i}(\boldsymbol{x}, t) \\
& =\epsilon(t) \dot{x}^{i}+\tilde{\eta}^{i}(\boldsymbol{x}, t) \\
\delta_{0} r^{a} & =\epsilon(t) \dot{r}^{a}+c_{b c}{ }^{a} \omega^{b}(\boldsymbol{x}, t) r^{c}+c_{b c}{ }^{a} \theta^{b}(\boldsymbol{x}, t) r^{c}+\sqrt{\frac{\mu}{k}} \xi^{a b}(\boldsymbol{x}, t) \delta_{b c} \dot{r}^{c} \\
& =\epsilon(t) \dot{r}^{a}+c_{b c}{ }^{a} \tilde{\theta}^{b}(\boldsymbol{x}, t) r^{c}+\sqrt{\frac{\mu}{k}} \xi^{a b}(\boldsymbol{x}, t) \delta_{b c} \dot{r}^{c},
\end{aligned}\right.
$$

(where we have set $\tilde{\eta}^{i}(\boldsymbol{x}, t) \equiv \epsilon^{i}(\boldsymbol{x}, t)+c_{j k}{ }^{l} \omega^{j}(\boldsymbol{x}, t) x^{k}-t v^{i}(\boldsymbol{x}, t)$ and $\tilde{\theta}^{b}(\boldsymbol{x}, t)=$ $\left.\theta^{b}(\boldsymbol{x}, t)+\omega^{b}(\boldsymbol{x}, t)\right)$. Instructed by the results obtained in Section $\mathbf{3}$, we know that the compensating $S U(3) \otimes U(1)$ Yang-Mills fields have to appear in the "internal" covariant derivative and in the "internal" metric. Furthermore, the dynamical component of the $S U(3) \otimes U(1)$ symmetry must be modified. Finally, from Section 4, we know in addition that, in gauging the Galilei part, a field $\Theta(t)$ must necessarily be introduced to take into account the redefinition of absolute time.

As a matter of fact, the required generalization can be achieved in the following way:

1) define the "internal" covariant derivative and "internal" metric as:

$$
\left\{\begin{aligned}
D r^{a} & =\dot{r}^{a}+\dot{x}^{k} B_{k}^{(b)}(\boldsymbol{x}, t) c_{b c}{ }^{a} r^{c}+B_{0}^{(b)}(\boldsymbol{x}, t) c_{b c}{ }^{a} r^{c} \\
\Xi^{a b}(\boldsymbol{x}, t) & =\delta^{a b}-\frac{1}{\Theta(t)} \sqrt{\frac{\mu}{k}}\left[\dot{x}^{k} B_{k}^{(a b)}(\boldsymbol{x}, t)-B_{0}^{(a b)}(\boldsymbol{x}, t)\right]
\end{aligned}\right.
$$


2) define the dynamical modified symmetry transformations as:

$$
\left\{\begin{array}{l}
\delta_{0} x^{i}=\epsilon(t) \dot{x}^{i}+\tilde{\eta}^{i}(\boldsymbol{x}, t) \\
\delta_{0} r^{a}=\epsilon(t) \dot{r}^{a}+c_{b c}{ }^{a} \tilde{\theta}^{b}(\boldsymbol{x}, t) r^{c}+\frac{1}{\Theta} \sqrt{\frac{\mu}{k}} \xi^{a b}(\boldsymbol{x}, t)\left|\Xi^{-1}\right|_{b c}(\boldsymbol{x}, t) \dot{r}^{c} .
\end{array}\right.
$$

3) write the final complete Lagrangian in the form:

$$
\begin{aligned}
\mathcal{L}_{G}= & \frac{M}{2 \Theta(t)}\left[g_{i j}(\boldsymbol{x}, t) \dot{x}^{i} \dot{x}^{j}+2 A_{i}(\boldsymbol{x}, t) \dot{x}^{i}+2 A_{0}(\boldsymbol{x}, t)\right] \\
& +\frac{\mu}{2 \Theta}\left|\Xi^{-1}\right|_{a b}(\boldsymbol{x}, t) D r^{a} D r^{b}-\frac{k \Theta}{2} \Xi^{a b}(\boldsymbol{x}, t) \delta_{a c} \delta_{b d} r^{c} r^{d} .
\end{aligned}
$$

Then, the complete transformation rules for the Yang-Mills fields are derived by requiring that the Action defined by the Lagrangian (5.4) be quasi-invariant under the maximal dynamical symmetry group gauged à la Utiyama, precisely in the form:

$$
\begin{aligned}
\delta_{0} \int d t \mathcal{L}_{G}=\int d t\left[\delta_{0} \mathcal{L}_{G}-\mathcal{L}_{G} \dot{\epsilon}\right] \\
=\int d t \frac{d}{d t}\left[-m g_{i j}(\boldsymbol{x}, t) v^{i}(\boldsymbol{x}, t) x^{j}\right] \\
+\int d t \frac{d}{d t}\left[\sqrt { \frac { \mu } { k } } \xi ^ { a b } ( \boldsymbol { x } , t ) \left(\frac{\mu}{2 \Theta}\left|\Xi^{-1}\right|_{a c}(\boldsymbol{x}, t) D r^{c}\left|\Xi^{-1}\right|_{b d}(\boldsymbol{x}, t) D r^{d}\right.\right. \\
\left.\left.-\frac{k \Theta}{2} \delta_{a c} \delta_{b d} r^{c} r^{d}\right)\right] .
\end{aligned}
$$

Clearly, the transformation rules of the gauge fields associated to the Galilei group and the dynamical symmetry $S U(3) \otimes U(1)$, when considered separately, have to be exactly those we have just found for the separate case of the gauged Galilei group (Section 4) and of the the gauged $S U(3) \otimes U(1)$ dynamical group (section 3). However, given the semi-direct product structure of the overall symmetry, the $S U(3) \otimes U(1)$ Yang-Mills fields must undergo additional transformations under the Galilei group.

In carrying out the calculation, it is essential to take into account the fact that we are imposing invariance of the Action with respect to transformations depending on the evolution parameter explicitly. Therefore, as shown by eq.(2.10), we have to consider the following relation between the pure field variation and the actual variation imposed on the Lagrangian: $\delta_{0} f(\boldsymbol{x}, t)=\delta f(\boldsymbol{x}, t)+\frac{d}{d t} f(\boldsymbol{x}, t) \dot{\epsilon}(t)$. Then, it follows that the compensating gauge fields corresponding to variations (5.3) satisfy the following transformation rules:

$$
\begin{aligned}
\hat{\delta}_{0}^{g} \Theta(t)= & \dot{\epsilon}(t) \Theta(t) \\
\hat{\delta}_{0}^{g} g_{i j}(\boldsymbol{x}, t)= & -\frac{\partial \tilde{\eta}^{k}(\mathbf{x}, t)}{\partial x^{i}} g_{k j}-\frac{\partial \tilde{\eta}^{k}(\mathbf{x}, t)}{\partial x^{i}} g_{k j} \\
\hat{\delta}_{0}^{g} A_{0}(\boldsymbol{x}, t)= & 2 \dot{\varepsilon} A_{0}-A_{i} \frac{\partial \tilde{\eta}^{i}}{\partial t}-\Theta \frac{\partial}{\partial t}\left[g_{i j} v^{i} x^{j}\right] \\
\hat{\delta}_{0}^{g} A_{i}(\boldsymbol{x}, t)= & \dot{\varepsilon} A_{i}-A_{j} \frac{\partial \tilde{\eta}^{j}}{\partial x^{i}}-g_{i j} \frac{\partial \tilde{\eta}^{j}}{\partial t}-\Theta \frac{\partial}{\partial x^{i}}\left[g_{j k} v^{j} x^{k}\right] . \\
\hat{\delta}_{0}^{g} B_{k}^{(a)}(\boldsymbol{x}, t)= & c_{b c}{ }^{a} \tilde{\theta}^{b}(\boldsymbol{x}, t) B_{k}^{(c)}(\boldsymbol{x}, t) \\
& +\xi^{c d}(\boldsymbol{x}, t)\left[c_{c e}{ }^{a} \delta_{d f} B_{k}^{(e f)}(\boldsymbol{x}, t)+c_{d e}{ }^{a} \delta_{c f} B_{k}^{(e f)}(\boldsymbol{x}, t)\right]
\end{aligned}
$$




$$
\begin{aligned}
& -\frac{\partial \tilde{\eta}^{i}}{\partial x^{k}} B_{i}^{(a)}(\boldsymbol{x}, t)-\frac{\partial \tilde{\theta}^{a}(\boldsymbol{x}, t)}{\partial x^{k}} \\
\hat{\delta}_{0}^{g} B_{0}^{(a)}(\boldsymbol{x}, t)= & c_{b c}{ }^{a} \tilde{\theta}^{b}(\boldsymbol{x}, t) B_{0}^{(c)}(\boldsymbol{x}, t) \\
& +\xi^{c d}(\boldsymbol{x}, t)\left[c_{c e}{ }^{a} \delta_{d f} B_{0}^{(e f)}(\boldsymbol{x}, t)+c_{d e}{ }^{a} \delta_{c f} B_{0}^{(e f)}(\boldsymbol{x}, t)\right] \\
& +\dot{\epsilon} B_{0}^{(a)}(\boldsymbol{x}, t)-\frac{\partial \tilde{\eta}^{i}}{\partial t} B_{i}^{(a)}(\boldsymbol{x}, t)-\frac{\partial \tilde{\theta}^{a}(\boldsymbol{x}, t)}{\partial t} \\
\hat{\delta}_{0}^{g} B_{k}^{(a b)}(\boldsymbol{x}, t)= & \tilde{\theta}^{c}(\boldsymbol{x}, t)\left[c_{c d}{ }^{a} B_{k}^{(d b)}(\boldsymbol{x}, t)+c_{c d}{ }^{b} B_{k}^{(d a)}(\boldsymbol{x}, t)\right] \\
& +\frac{1}{4} \xi^{c d}(\boldsymbol{x}, t)\left[c_{e c}{ }^{a} \delta_{d}^{b}+c_{e c}{ }^{b} \delta_{d}^{a}+c_{e d}{ }^{a} \delta_{c}^{b}+c_{e d}{ }^{b} \delta_{c}^{a}\right] B_{k}^{(e)}(\boldsymbol{x}, t) \\
& -\frac{\partial \tilde{\eta}^{i}}{\partial x^{k}} B_{i}^{(a b)}(\boldsymbol{x}, t)-\frac{\partial \xi^{a b}(\boldsymbol{x}, t)}{\partial x^{k}} \\
\hat{\delta}_{0}^{g} B_{0}^{(a b)}(\boldsymbol{x}, t)= & \tilde{\theta}^{c}(\boldsymbol{x}, t)\left[c_{c d}{ }^{a} B_{0}^{(d b)}(\boldsymbol{x}, t)+c_{c d}^{b} B_{0}^{(d a)}(\boldsymbol{x}, t)\right] \\
& +\frac{1}{4} \xi^{c d}(\boldsymbol{x}, t)\left[c_{e c}{ }^{a} \delta_{d}^{b}+c_{e c}{ }^{b} \delta_{d}^{a}+c_{e d}{ }^{a} \delta_{c}^{b}+c_{e d}{ }^{b} \delta_{c}^{a}\right] B_{0}^{(e)}(\boldsymbol{x}, t) \\
& +\dot{\epsilon} B_{0}^{(a b)}(\boldsymbol{x}, t)-\frac{\partial \tilde{\eta}^{i}}{\partial t} B_{i}^{(a b)}(\boldsymbol{x}, t)-\frac{\partial \xi^{a b}(\boldsymbol{x}, t)}{\partial t}
\end{aligned}
$$

In conclusion, we see from equations (5.2) and (5.4), that we have to take into account with the following gauge fields:

1) either the Euclidean metric $g_{i j}(\boldsymbol{x}, t)\left(\left(g^{-1}\right)^{i j}(\boldsymbol{x}, t)\right)$ or the dreibein $\boldsymbol{E}_{i}^{a}(\boldsymbol{x}, t)$ $\left(\boldsymbol{H}_{a}^{i}(\boldsymbol{x}, t)\right)$

2) the time reparameterization field $\Theta(t)$;

3) the inertial-gravitational fields $A_{i}(\boldsymbol{x}, t)$ and $A_{0}(\boldsymbol{x}, t)$;

4) the "spin connection" fields $B_{i}^{(a)}(\boldsymbol{x}, t), B_{0}^{(a)}(\boldsymbol{x}, t)$, which generate the internal covariant derivative;

5) the quadrupole and internal energy Yang-Mills fields $B_{i}^{(a b)}(\boldsymbol{x}, t), B_{0}^{(a b)}(\boldsymbol{x}, t)$, which generate the internal metric $\Xi^{r s}(\boldsymbol{x}, t)$,

Finally, let us remark that, going back to the Hamiltonian picture, we would find that the above procedure corresponds to minimal substitution rules for energy and canonical momenta given by:

$$
\begin{aligned}
P_{i} & \mapsto \mathcal{P}_{a}=\mathbf{H}_{a}^{i}(\boldsymbol{x}, t)\left[P_{i}-\frac{M}{\Theta} A_{i}(\boldsymbol{x}, t)-B_{i}^{(a)}(\boldsymbol{x}, t) S_{a}-B_{i}^{(a b)}(\boldsymbol{x}, t) N_{a b}\right] \\
E & \mapsto \mathcal{E}=\frac{1}{\Theta}\left[E-\frac{M}{\Theta} A_{0}(\boldsymbol{x}, t)-B_{0}^{(a)}(\boldsymbol{x}, t) S_{a}-B_{0}^{(a b)}(\boldsymbol{x}, t) N_{a b}\right]
\end{aligned}
$$

while, for the Hamiltonian itself, we would find:

$$
H \mapsto \mathcal{H}=\Theta(t) H[\mathcal{P}]-\frac{M}{\Theta} A_{0}(\boldsymbol{x}, t)-B_{0}^{(a)}(\boldsymbol{x}, t) \bar{S}_{a}-B_{0}^{(a b)}(\boldsymbol{x}, t) \bar{N}_{a b}
$$

How all this can work will become clear presently in approaching the whole problem within the multi-temporal picture. 
It is interesting to rewrite the final Lagrangian (5.4) in terms of the individual coordinates of the constituents

$$
\begin{aligned}
\mathcal{L}_{G}= & \frac{m_{1}}{2 \Theta(t)}\left[\frac{m_{1}}{M} g_{i j}(\boldsymbol{x}, t)+\frac{\mu}{m_{1}}\left|\Xi^{-1}\right|_{i j}(\boldsymbol{x}, t)\right] \dot{x}_{1}^{i} \dot{x}_{1}^{j} \\
& +\frac{m_{2}}{2 \Theta(t)}\left[\frac{m_{2}}{M} g_{i j}(\boldsymbol{x}, t)+\frac{\mu}{m_{2}}\left|\Xi^{-1}\right|_{i j}(\boldsymbol{x}, t)\right] \dot{x}_{2}^{i} \dot{x}_{2}^{j} \\
& +\frac{\mu}{\Theta(t)}\left[g_{i j}(\boldsymbol{x}, t)-\left|\Xi^{-1}\right|_{i j}(\boldsymbol{x}, t)\right] \dot{x}_{1}^{i} \dot{x}_{2}^{j} \\
& +\frac{m_{1}}{\Theta}\left[\dot{x}_{1}^{k} A_{k}\left(\boldsymbol{x}_{1}, t\right)+A_{0}\left(\boldsymbol{x}_{1}, t\right)\right]+\frac{m_{2}}{\Theta}\left[\dot{x}_{2}^{k} A_{k}\left(\boldsymbol{x}_{2}, t\right)+A_{0}\left(\boldsymbol{x}_{2}, t\right)\right] \\
& +\sum_{\alpha=1}^{\infty} \frac{\mu^{\alpha}}{\Theta}\left[\left(\frac{1}{m_{2}}\right)^{\alpha}\left(\dot{x}_{2}^{k} A_{k, i_{1} \cdots i_{\alpha}}\left(\boldsymbol{x}_{2}, t\right)+A_{0, i_{1} \cdots i_{\alpha}}\left(\boldsymbol{x}_{2}, t\right)\right)\right. \\
& \left.\quad+\left(\frac{-1}{m_{1}}\right)^{\alpha}\left(\dot{x}_{1}^{k} A_{k, i_{1} \cdots i_{\alpha}}\left(\boldsymbol{x}_{1}, t\right)+A_{0, i_{1} \cdots i_{\alpha}}\left(\boldsymbol{x}_{1}, t\right)\right)\right] \\
& \quad \cdot\left(x_{1}^{i_{1}}-x_{2}^{i_{1}}\right) \cdots\left(x_{1}^{i_{\alpha}}-x_{2}^{i_{\alpha}}\right) \\
& +\frac{\mu}{\Theta}\left|\Xi^{-1}\right|_{a b}(\boldsymbol{x}, t) \dot{r}^{a} \epsilon_{e f}^{b}\left[\dot{x}^{k} B_{k}^{(e)}(\boldsymbol{x}, t)+B_{0}^{(e)}(\boldsymbol{x}, t)\right]\left(x_{1}^{f}-x_{2}^{f}\right) \\
& +\frac{\mu}{2 \Theta}\left|\Xi^{-1}\right|_{a b}(\boldsymbol{x}, t) \epsilon_{e f}^{a}\left[\dot{x}^{k} B_{k}^{(e)}(\boldsymbol{x}, t)+B_{0}^{(e)}(\boldsymbol{x}, t)\right]\left(x_{1}^{f}-x_{2}^{f}\right) \\
& \quad \epsilon_{g h}^{b}\left[\dot{x}^{k} B_{k}^{(g)}(\boldsymbol{x}, t)+B_{0}^{(g)}(\boldsymbol{x}, t)\right]\left(x_{1}^{h}-x_{2}^{h}\right) \\
& -\frac{k \Theta}{2} \Xi^{a b}(\boldsymbol{x}, t) \delta_{a c} \delta_{b d}\left(x_{1}^{c}-x_{2}^{c}\right)\left(x_{1}^{d}-x_{2}^{d}\right) \cdot \quad,
\end{aligned}
$$

where the expressions of the fields calculated in $\boldsymbol{x}$ have to be reexpressed as multipolar series in the relative variable $\boldsymbol{r}^{i}=x_{1}^{i}-x_{2}^{i}$, as, for example:

$$
\begin{aligned}
& \dot{x}^{k} B_{k}^{(a)}(\boldsymbol{x}, t)+ B_{0}^{(a)}(\boldsymbol{x}, t) \\
&= \frac{m_{1}}{M}\left[\dot{x}_{1}^{k} B_{k}^{(a)}\left(\boldsymbol{x}_{1}, t\right)+B_{0}^{(a)}\left(\boldsymbol{x}_{1}, t\right)\right]+\frac{m_{2}}{M}\left[\dot{x}_{2}^{k} B_{k}^{(a)}\left(\boldsymbol{x}_{2}, t\right)+B_{0}^{(a)}\left(\boldsymbol{x}_{2}, t\right)\right] \\
&+\sum_{\alpha=1}^{\infty} \frac{\mu^{\alpha}}{\Theta}\left[\left(\frac{1}{m_{2}}\right)^{\alpha}\left(\dot{x}_{2}^{k} B_{k, i_{1} \cdots i_{\alpha}}^{(a)}\left(\boldsymbol{x}_{2}, t\right)+B_{0, i_{1} \cdots i_{\alpha}}^{(a)}\left(\boldsymbol{x}_{2}, t\right)\right)\right. \\
&\left.+\left(\frac{-1}{m_{1}}\right)^{\alpha}\left(\dot{x}_{1}^{k} B_{k, i_{1} \cdots i_{\alpha}}^{(a)}\left(\boldsymbol{x}_{1}, t\right)+B_{0, i_{1} \cdots i_{\alpha}}^{(a)}\left(\boldsymbol{x}_{1}, t\right)\right)\right] \\
& \cdot\left(x_{1}^{i_{1}}-x_{2}^{i_{1}}\right) \cdots\left(x_{1}^{i_{\alpha}}-x_{2}^{i_{\alpha}}\right)
\end{aligned}
$$

It is remarkable that our constructive method based on Noether's theorem and Utiyama procedure leads to a non-local coupling to the individual constituents of the dynamical system even if the masses (sources of the Galilean gravitational fields) are localized at their positions.

\section{The 2-times harmonic oscillator with center-of-mass}

A description of the non-relativistic harmonic oscillator in a 16-dimensional phase space coordinatized by $t_{u}, E_{u}, \overrightarrow{x_{u}}, \overrightarrow{p_{u}}\left(u=1,2 ;\left\{t_{u}, E_{v}\right\}=-\delta_{u v},\left\{x_{u}^{i}, p_{v j}\right\}=\delta_{u v} \delta_{j}^{i}\right)$ 
can be easily obtained by studying the non-relativistic limit of the Todorov-KomarDroz Vincent relativistic harmonic oscillator. The latter is described by two first-class constraints in the 16 variables $x_{u}^{\mu}, p_{u \mu}$, and by a mutual action-at-a-distance interaction that is instantaneous in the center-of-mass frame (see ref [4]). The non-relativistic counterpart of this system is described by the following two first-class constraints (the Hamiltonian is zero)

$$
\left\{\begin{array}{l}
\bar{\chi}_{a}=E_{a}-\frac{1}{2 m_{a}}\left(\delta^{i j} p_{a i} p_{a j}+\mu k \delta_{i j} \rho^{i} \rho^{j}\right) \simeq 0 \quad, \quad a=1,2 \\
\left\{\bar{\chi}_{1}, \bar{\chi}_{2}\right\}=0,
\end{array}\right.
$$

where $\rho^{i}$ is defined by

$$
\rho^{i}=r^{i}-\frac{t_{1}-t_{2}}{M} \delta^{i j} P_{j},
$$

and $P_{i}=p_{1 i}+p_{2 i}$.

Besides eqs.(2.2) and (2.5), we introduce the following definitions:

$$
\left\{\begin{array} { r l } 
{ t } & { = \frac { m _ { 1 } t _ { 1 } + m _ { 2 } t _ { 2 } } { M } } \\
{ t _ { R } } & { = t _ { 1 } - t _ { 2 } } \\
{ E } & { = E _ { 1 } + E _ { 2 } } \\
{ E _ { R } } & { = \frac { m _ { 2 } E _ { 1 } - m _ { 1 } E _ { 2 } } { M } }
\end{array} \left\{\begin{array}{rl}
t_{1} & =t+\frac{m_{2}}{M} t_{R} \\
t_{2} & =t-\frac{m_{2}}{M} t_{R} \\
E_{1} & =\frac{m_{1}}{M} E+E_{r} \\
E_{2} & =\frac{m_{2}}{M} E-E_{r}
\end{array}\right.\right.
$$

and, for the sake of simplicity, the following linear combinations of the $\bar{\chi}_{u}$ 's:

$$
\left\{\begin{aligned}
\bar{\chi}_{+} & =\bar{\chi}_{1}+\bar{\chi}_{2}=E-\frac{1}{2 \mu} \delta^{i j} \pi_{i} \pi_{j}+\frac{k}{2} \delta_{i j} \rho^{i} \rho^{j} \simeq 0 \\
\bar{\chi}_{-} & =\frac{m_{2} \bar{\chi}_{1}-m_{1} \bar{\chi}_{2}}{M} \\
& \left.=E_{r}-\frac{1}{M} \delta^{i j} P_{i} \pi_{j}-\frac{m_{2}-m_{1}}{M}\left(\frac{1}{2 \mu} \delta^{i j} \pi_{i} \pi_{j}+\frac{k}{2} \delta_{i j} \rho^{i} \rho^{j}\right)\right) \simeq 0 .
\end{aligned}\right.
$$

For $t_{R}=0$, one has $\bar{\chi}_{+}=E-\bar{H} \simeq 0$ and, correspondingly, the usual Hamiltonian formulation for a non-relativistic system invariant under time reparameterization.

The Galilei generators in the Hamiltonian picture are now:

$$
\left\{\begin{aligned}
E & =E_{1}+E_{2} \\
P_{i} & =p_{1 i}+p_{2 i} \\
J_{i} & =c_{i j}{ }^{k} x_{1}^{j} p_{1 k}+c_{i j}{ }^{k} x_{2}^{j} p_{2 k}=c_{i j}{ }^{k} x^{j} P_{k}+c_{i j}{ }^{k} r^{j} \pi_{k} \\
K_{i} & =m_{1} \delta_{i j} x_{1}^{j}-t_{1} p_{1 i}+m_{2} \delta_{i j} x_{2}^{j}-t_{1} p_{2 i}=M \delta_{i j} x^{j}-t P_{i}-t_{R} \pi_{i} .
\end{aligned}\right.
$$

Of course, these generators are constants of the motion, having zero Poisson brackets with both the constraints and, consequently, with the Dirac Hamiltonian $H_{D}=$ $\lambda_{1} \chi_{1}+\lambda_{2} \chi_{2}$.

The generators of the $S U(3) \otimes U(1)$ dynamical symmetry are now:

$$
\left\{\begin{aligned}
\tilde{\bar{S}}_{a} & =c_{a b}^{c} \rho^{b} \pi_{c} \\
\tilde{\bar{N}}_{a b} & =\frac{1}{2 \sqrt{\mu k}} \pi_{a} \pi_{b}+\frac{\sqrt{\mu k}}{2} \delta_{a c} \delta_{b d} \rho^{c} \rho^{d} .
\end{aligned}\right.
$$


They satisfy the algebra (2.19) and have zero Poisson brackets with the two constraints $\bar{\chi}_{ \pm}$, because $\left\{\vec{\rho}, E_{R}-\frac{\vec{p} \cdot \pi}{M}\right\}=0$. For $t_{R}=0$, they assume the expression (2.17), (2.18) of the one-time theory. Again, we have the structure of a semi-direct product of the two algebras (6.5) and (6.6), since eqs. (2.24) are still valid.

The generator $G_{U}=\theta^{a} \tilde{\bar{S}}_{a}+\xi^{a b} \tilde{\bar{N}}_{a b}$ induces the variation

$$
\left\{\begin{array}{l}
\bar{\delta} x^{i}=\frac{t_{r}}{M} \delta^{i j} c_{j k}{ }^{l} \theta^{k} \pi_{l}-\frac{t_{r}}{M} \sqrt{\mu k} \xi^{i j} \delta_{j k} \rho^{k} \\
\bar{\delta} \rho^{a}=c_{b c} \theta^{a} \rho^{c}+\frac{1}{\sqrt{\mu k}} \xi^{a b} \pi_{b} .
\end{array}\right.
$$

Now, since $\left\{x^{i}, \rho^{j}\right\}=-\frac{t_{R}}{M} \delta^{i j} \neq 0$, the center-of-mass coordinates invariant under the transformation of the dynamical symmetry are

$$
\tilde{x}^{i}=x^{i}-t_{R} \frac{\delta^{i j} \pi_{j}}{M}
$$

Then, performing the canonical transformation

$$
\left[\begin{array}{lll}
t & , & E \\
t_{R} & , & E_{R} \\
p_{i} & , & x^{i} \\
\pi_{i} & , & r^{i}
\end{array}\right] \longmapsto\left[\begin{array}{lll}
t & , & E \\
t_{R} & , & \tilde{E}_{R}=E_{R}-\frac{\delta^{i j} p_{i} \pi_{j}}{M} \\
p_{i} & , & \tilde{x}^{i}=x^{i}-t_{R} \frac{\delta^{i j} p_{j}}{M} \\
\pi_{i} & , & \rho^{i}=r^{i}-t_{R} \frac{\delta^{i j} \pi_{j}}{M}
\end{array}\right],
$$

the canonical generators, associated to Galilei boosts and rotations, become $\bar{K}_{i}=M \delta_{i j} \tilde{x}^{j}-t p_{i}$ and $\bar{J}_{i}=c_{i j}{ }^{k} \tilde{x}^{j} p_{k}+c_{i j}{ }^{k} \rho^{j} \pi_{k}$, while the constraint $\bar{\chi}_{-} \simeq 0$ becomes $\tilde{\bar{\chi}}_{-}=\tilde{E}_{R}-\frac{m_{1}-m_{2}}{2 M \mu \simeq 0}\left(\delta^{a b} \pi_{a} \pi_{b}+\mu k \delta_{a b} \rho^{a} \rho^{b}\right) \simeq 0$ and the constraint $\tilde{\bar{\chi}}_{+} \simeq 0$ is left unchanged.

As shown in [4], the constraints (6.1) follow from the singular Lagrangian

$$
\begin{aligned}
\mathcal{L}^{\star}(\tau)= & {\left[1+\frac{k t_{R}^{2}}{M^{2}} \frac{m_{1} m_{2}}{t_{1}^{\prime} t_{2}^{\prime}}\left(\frac{t_{1}^{\prime}}{m_{1}}+\frac{t_{2}^{\prime}}{m_{2}}\right)\right]^{-1} . } \\
& \cdot\left\{\frac{m_{1}}{2 t_{1}^{\prime}} \delta_{i j} V_{1}^{i} V_{1}^{j}+\frac{m_{2}}{2 t_{2}^{\prime}} \delta_{i j} V_{2}^{i} V_{2}^{j}\right. \\
& \left.+\frac{k t_{R}^{2}}{M^{2}} \frac{m_{1} m_{2}}{t_{1}^{\prime} t_{2}^{\prime}}\left(\frac{t_{1}^{\prime}}{m_{1}}+\frac{t_{2}^{\prime}}{m_{2}}\right) \delta_{i j}\left(V_{1}^{i}-V_{2}^{i}\right)\left(V_{1}^{j}-V_{2}^{j}\right)\right\} \\
& -\frac{k}{2}\left(\frac{t_{1}^{\prime}}{m_{1}}+\frac{t_{2}^{\prime}}{m_{2}}\right) \delta_{i j} \rho_{i} \rho_{j},
\end{aligned}
$$

where $f^{\prime}(\tau)=\frac{d}{d \tau} f(\tau)$ and

$$
V_{a}^{i}=x_{a}^{i}+\frac{k t_{R}}{M}\left(\frac{t_{1}^{\prime}}{m_{1}}+\frac{t_{2}^{\prime}}{m_{2}}\right) .
$$

The Action $\mathcal{A}^{\star}=\int d \tau \mathcal{L}^{\star}(\tau)$ clearly reduces to the Action generated by the Lagrangian (2.1) for $t_{R}=0$, i.e. $t_{1}=t_{2}=t$.

\section{Gauging the $S U(3) \otimes U(1)$ group in the two-times theory}

Having the relativistic case in view ("Todorov-Komar-Droz Vincent" harmonic oscillator [8, 9]), it is instrumental to discuss also the two-times formulation. 
Once the constraints $\bar{\chi}_{ \pm}$are expressed as $\tilde{\bar{\chi}}_{ \pm}$, in terms of the natural canonical basis $\left[t, E, t_{R}, \tilde{E}_{R}, \tilde{\boldsymbol{x}}, \boldsymbol{P}, \boldsymbol{\rho}, \boldsymbol{\pi}\right]$ (see eq. (6.9)), it is apparent that they actually depend only on $E, \tilde{E}_{R}$ and the $S U(3) \otimes U(1)$ invariant $\delta^{i j} \pi_{i} \pi_{j}+k \delta_{i j} \rho^{i} \rho^{j}$. Thus, their $S U(3) \otimes U(1)$ invariance is manifest. Consequently, we will now require that $\tilde{\bar{\chi}}_{ \pm}$ and the Dirac Hamiltonian be modified in such a way that they remain invariant also under the transformations generated by the generators $G_{U} \equiv \theta^{i} \tilde{\bar{S}}_{i}+\xi^{i j} \tilde{\bar{N}}_{i j}$, localized at new "event" $[\tilde{\boldsymbol{x}}, t]$. Now under $G_{U}(\tilde{\boldsymbol{x}}, t) \equiv \theta^{i}(\tilde{\boldsymbol{x}}, t) \tilde{\bar{S}}_{i}+\xi^{i j}(\tilde{\boldsymbol{x}}, t) \tilde{\bar{N}}_{i j}$, besides $\bar{\delta} \boldsymbol{\rho} \neq 0$, $\bar{\delta} \boldsymbol{\pi} \neq 0$, we have also $\bar{\delta} \boldsymbol{P} \neq 0$, (as in Eq. 3.13 ) ) and $\delta E \neq 0$, while the variations of the $S U(3) \otimes U(1)$ generators maintain the form (3.4) with $\boldsymbol{r}$ replaced by $\boldsymbol{\rho}$. Then, in analogy to eqs.(3.29) and (3.30) of Section 3, the invariance is recovered by adopting the minimal coupling

$$
\begin{aligned}
P_{i} & \mapsto \mathcal{P}_{i}=P_{i}-B_{i}^{(a)}(\tilde{\boldsymbol{x}}, t) S_{a}-B_{i}^{(a b)}(\tilde{\boldsymbol{x}}, t) N_{a b} \\
E & \mapsto \mathcal{E}=E-B_{0}^{(a)}(\tilde{\boldsymbol{x}}, t) S_{a}-B_{0}^{(a b)}(\tilde{\boldsymbol{x}}, t) N_{a b},
\end{aligned}
$$

and the requirement that the Yang-Mills fields $B_{k}^{(a)}, B_{0}^{(a)}, B_{k}^{(a b)}, B_{0}^{(a b)}$ transform as in eqs. (3.26) with $\vec{x}$ replaced by $\overrightarrow{\tilde{x}}$.

Therefore, we get the invariant constraints

$$
\left\{\begin{array}{cc}
\bar{\chi}_{+}^{S U(3) \otimes U(1)}= & E-B_{0}^{(a)}(\tilde{\boldsymbol{x}}, t) S_{a}-B_{0}^{(a b)}(\tilde{\boldsymbol{x}}, t) N_{a b} \\
& -\frac{1}{2 M} \delta^{i j}\left(P_{i}-B_{i}^{(a)}(\tilde{\boldsymbol{x}}) \tilde{\bar{S}}_{a}-B_{i}^{(a b)}(\tilde{\boldsymbol{x}}) \tilde{\bar{N}}_{a b}\right) \\
& \left(P_{j}-B_{j}^{(a)}(\tilde{\boldsymbol{x}}) \tilde{\bar{S}}_{a}-B_{j}^{(a b)}(\tilde{\boldsymbol{x}}) \tilde{\bar{N}}_{a b}\right) \\
& -\frac{1}{2 \mu}\left(\delta^{i j} \pi_{i} \pi_{j}+\mu k \delta_{i j} \rho^{i} \rho^{j}\right) \simeq 0 \\
\bar{\chi}_{-}^{S U(3) \otimes U(1)}= & \tilde{E}_{R}-\frac{m_{1}-m_{2}}{2 M \mu}\left(\delta^{i j} \pi_{i} \pi_{j}+\mu k \delta_{i j} \rho^{i} \rho^{j}\right) \simeq 0
\end{array}\right.
$$

and we still have the integrability condition of the multi-temporal theory:

$$
\left\{\bar{\chi}_{+}^{S U(3) \otimes U(1)}, \bar{\chi}_{-}^{S U(3) \otimes U(1)}\right\}=0
$$

Were we able to perform the inverse Legendre transformation independently of the specific functional form of the external gauge fields, we could find a putative Lagrangian $\mathcal{L}_{S U(3) \otimes U(1)}^{\star}$ which would be the counterpart of the free Lagrangian $\mathcal{L}^{\star}$ (6.10) with non-minimally coupled external fields. Yet, this is not the case as it is clear from the fact that the above procedure would amount to solving the following 
equations for $\boldsymbol{P}, \boldsymbol{\pi}, \lambda_{+}(\tau), \lambda_{-}(\tau)$, as function of $\frac{d x^{i}}{d \tau}, \frac{d t}{d \tau}, \frac{d r^{a}}{d \tau}, \frac{d t_{R}^{i}}{d \tau}$ :

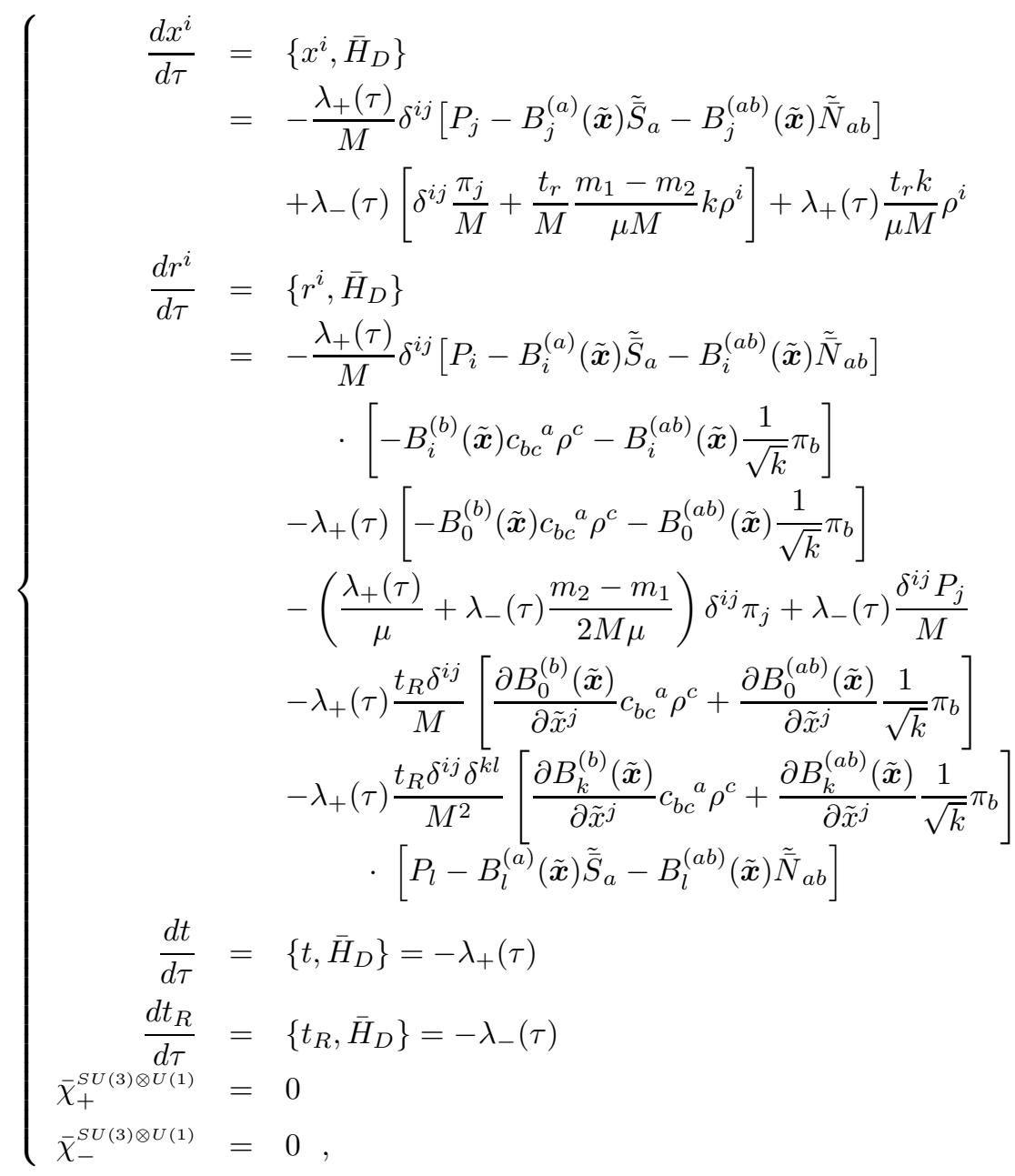

(where $\bar{H}_{D}=\lambda_{+} \bar{\chi}_{+}^{S U(3) \otimes U(1)}+\lambda_{-} \bar{\chi}_{-}^{S U(3) \otimes U(1)}$ is the Dirac's Hamiltonian). In particular, one would have to solve for, e.g., the variable $\boldsymbol{\pi}$, which is itself an argument of the gauge fields through $\tilde{\boldsymbol{x}}$.

On the other hand, a Lagrangian description of the gauge-invariant system can be obtained in a natural and simpler way by fully resorting to new position and energy 
particle variables $\left[\tilde{x}_{1}^{i}, \tilde{x}_{2}^{i}, \tilde{E}_{1}, \tilde{E}_{2}\right]$ defined by

$$
\left\{\begin{array} { r l } 
{ t _ { 1 } } & { = t + \frac { m _ { 2 } } { M } t _ { R } } \\
{ \tilde { E } _ { 1 } } & { = E _ { 1 } - \frac { \delta ^ { i j } p _ { 1 } \pi _ { j } } { M } } \\
{ t _ { 2 } } & { = t - \frac { m _ { 2 } } { M } t _ { R } } \\
{ \tilde { E } _ { 2 } } & { = E _ { 2 } + \frac { \delta ^ { i j } p _ { 1 } \pi _ { j } } { M } } \\
{ \tilde { x } _ { 1 } ^ { i } } & { = \tilde { x } ^ { i } + \frac { m _ { 2 } } { M } \rho ^ { i } } \\
{ p _ { 1 i } } & { = \frac { m _ { 1 } } { M } P _ { i } + \pi _ { i } } \\
{ \tilde { x } _ { 2 } ^ { i } } & { = \tilde { x } ^ { i } - \frac { m _ { 1 } } { M } \rho ^ { i } } \\
{ p _ { 2 i } } & { = \frac { m _ { 2 } } { M } P _ { i } - \pi _ { i } }
\end{array} \quad \left\{\begin{array}{rl}
t & =\frac{m_{1} t_{1}+m_{2} t_{2}}{M} \\
t_{R} & =t_{1}-t_{2} \\
\tilde{E}_{R} & =\frac{m_{2} \tilde{E}_{1}-m_{1} \tilde{E}_{2}}{M} \\
\tilde{x}^{i} & =\frac{m_{1} \tilde{x}_{1}^{i}+m_{2} \tilde{x}_{2}^{i}}{M} \\
p_{i} & =p_{1 i}+p_{2 i} \\
\rho^{i} & =\tilde{x}_{1}^{i}-\tilde{x}_{2}^{i} \\
\pi_{i} & =\frac{m_{2} p_{1 i}-m_{1} p_{2 i}}{M}
\end{array}\right.\right.
$$

First of all, this is easily done for the uncoupled system. Actually, the original constraints (6.1) can be rewritten as:

$$
\left\{\begin{array}{l}
\bar{\chi}_{+}=\tilde{\bar{\chi}}_{1}+\tilde{\bar{\chi}}_{2}=E-\frac{1}{2 M} \delta^{i j} P_{i} P_{j}-\frac{1}{2 \mu}\left(\delta^{i j} \pi_{i} \pi_{j}+k \delta_{i j} \rho^{i} \rho^{j}\right) \simeq 0 \\
\bar{\chi}_{-}=\frac{m_{2} \tilde{\bar{\chi}}_{1}-m_{1} \tilde{\bar{\chi}}_{2}}{M}=\tilde{E}_{R}-\frac{m_{2}-m_{1}}{2 \mu M}\left(\delta^{i j} \pi_{i} \pi_{j}+k \delta_{i j} \rho^{i} \rho^{j}\right) \simeq 0
\end{array}\right.
$$

or

$$
\left\{\begin{array}{l}
\left.\tilde{\bar{\chi}}_{u}=\tilde{E}_{u}-\frac{1}{2 m_{u}}\left(\delta^{i j} p_{u i} p_{u j}+\mu k \delta_{i j} \rho^{i} \rho^{j}\right)\right) \simeq 0 \quad u=1,2 \\
\left\{\bar{\chi}_{1}, \bar{\chi}_{2}\right\}=0
\end{array}\right.
$$

Performing the inverse Legendre transformation, one obtains a Lagrangian dependent on $\left[t_{u}(\tau), t_{u}^{\prime}(\tau), \tilde{\boldsymbol{x}}_{u}^{i}(\tau), \tilde{\boldsymbol{x}}_{u}^{\prime i}(\tau)\right]$ or, what is the same, $\left[t_{u}(\tau), t_{a}^{\prime}(\tau), \tilde{\boldsymbol{x}}^{i}(\tau), \tilde{\boldsymbol{x}}^{\prime i}(\tau), \boldsymbol{\rho}(\tau), \boldsymbol{\rho}^{\prime}(\tau)\right]$ :

$$
\mathcal{L}^{\star}=\frac{M}{2} \frac{\delta_{i j} \tilde{x}^{\prime} \tilde{x}^{\prime j}}{t^{\prime}}+\frac{\mu}{2} \frac{\delta_{a b} \rho^{\prime a} \rho^{\prime b}}{t^{\prime}+\frac{m_{2}-m_{1}}{M} T^{\prime} R}-\frac{k}{2}\left(t^{\prime}+\frac{m_{2}-m_{1}}{M} T_{R}^{\prime}\right) \delta_{a b} \rho^{a} \rho^{b}
$$

On the other hand, the constraints of the coupled system (given by eq. (7.2)) determine 
the equations of motion

$$
\left\{\begin{aligned}
\tilde{x}^{\prime i}= & \left\{\tilde{x}^{i}, \bar{H}_{D}\right\} \\
= & -\frac{\lambda_{+}(\tau)}{M} \delta^{i j}\left(P_{j}-B_{j}^{(a)}(\tilde{\boldsymbol{x}}) \tilde{\bar{S}}_{a}-B_{j}^{(a b)}(\tilde{\boldsymbol{x}}) \tilde{\bar{N}}_{a b}\right) \\
\rho^{\prime a}= & \left\{\rho^{a}, \bar{H}_{D}\right\} \\
= & -\frac{\lambda_{+}(\tau)}{M} \delta^{i j}\left(P_{i}-B_{i}^{(a)}(\tilde{\boldsymbol{x}}) \tilde{\bar{S}}_{a}-B_{i}^{(a b)}(\tilde{\boldsymbol{x}}) \tilde{\bar{N}}_{a b}\right) \\
& \left(-B_{i}^{(b)}(\tilde{\boldsymbol{x}}) c_{b c}{ }^{a} \rho^{c}-B_{i}^{(a b)}(\tilde{\boldsymbol{x}}) \frac{1}{\sqrt{\mu k}} \pi_{b}\right) \\
& -\lambda_{+}(\tau)\left(-B_{0}^{(b)}(\tilde{\boldsymbol{x}}) c_{b c}{ }^{a} \rho^{c}-B_{0}^{(a b)}(\tilde{\boldsymbol{x}}) \frac{1}{\sqrt{\mu k}} \pi_{b}\right) \\
& -\left(\frac{\lambda_{+}(\tau)}{\mu}+\lambda_{-}(\tau) \frac{m_{2}-m_{1}}{2 M \mu}\right) \delta^{i j} \pi_{j} \\
t^{\prime}= & \left\{t, \bar{H}_{D}\right\}=-\lambda_{+} \\
t^{\prime}= & \left\{t_{R}, \bar{H}_{D}\right\}=-\lambda_{-}(\tau) \\
\bar{\chi}_{+}^{S U(3) \otimes U(1)}= & 0 \\
\bar{\chi}_{-}^{S U(3) \otimes U(1)}= & 0,
\end{aligned}\right.
$$

which can be explicitly solved for $\boldsymbol{P}, \quad \boldsymbol{\pi}, \lambda_{+}(\tau), \lambda_{-}(\tau)$ as functions of $\left[t_{u}(\tau), t_{u}^{\prime}(\tau), \tilde{\boldsymbol{x}}_{u}^{i}(\tau), \tilde{\boldsymbol{x}}_{u}^{\prime i}(\tau)\right]$

Thus, the inverse Legendre transformation can be carried out. As a matter of fact, by defining, in analogy to what done in section $\mathbf{3}$, the quantities

$$
\begin{aligned}
& D \rho^{a} \quad \equiv \rho^{a}+c_{b c}{ }^{a}\left(\tilde{x}^{\prime k} B_{k}^{(b)}(\tilde{\boldsymbol{x}}, t)+t^{\prime} B_{0}^{(b)}(\tilde{\boldsymbol{x}}, t)\right) \rho^{c} \\
& \tilde{\Xi}^{a b}\left(\tilde{\boldsymbol{x}}, t, t_{R}\right) \equiv \delta^{a b}+\frac{\mu}{\sqrt{\mu k}} \frac{1}{t^{\prime}+\frac{m_{2}-m_{1}}{M} t^{\prime}{ }_{R}}\left(\tilde{x}^{\prime k} B_{k}^{(a b)}(\tilde{\boldsymbol{x}}, t)+t^{\prime} B_{0}^{(a b)}(\tilde{\boldsymbol{x}}, t)\right),
\end{aligned}
$$

so that

$$
\tilde{\Xi}^{a b}\left(\tilde{\boldsymbol{x}}, t, t_{R}\right) \pi_{j}=\mu \frac{1}{t^{\prime}+\frac{m_{2}-m_{1}}{M} t_{R}^{\prime}} D \rho^{i}
$$

it follows

$$
\left\{\begin{aligned}
\lambda_{+} & =-t^{\prime} \\
\lambda_{-} & =-t^{\prime}{ }_{R} \\
\pi_{a} & =\mu \frac{1}{t^{\prime}+\frac{m_{2}-m_{1}}{M} t_{R}^{\prime}}\left|\Xi^{-1}\left(\tilde{\boldsymbol{x}}, t, t_{R}\right)\right|_{a b} D \rho^{b} \\
P_{i} & =M \delta_{i j} \tilde{x}^{\prime j}+B_{i}^{(a)}(\tilde{\boldsymbol{x}}) \tilde{\bar{S}}_{a}+B_{i}^{(a b)}(\tilde{\boldsymbol{x}}) \tilde{\bar{N}}_{a b}
\end{aligned}\right.
$$

In conclusion, the Lagrangian of the coupled system, $\tilde{\mathcal{L}}_{S U(3) \otimes U(1)}^{\star}$, is given by:

$$
\begin{aligned}
\tilde{\mathcal{L}}_{S U(3) \otimes U(1)}^{\star}= & \frac{M}{2} \frac{\delta_{i j} \tilde{x}^{\prime} \tilde{x}^{\prime j}}{t^{\prime}}+\frac{\mu}{2} \frac{\left|\Xi^{-1}\left(\tilde{\boldsymbol{x}}, t, t_{R}\right)\right|_{a b} D \rho^{a} D \rho^{b}}{t^{\prime}+\frac{m_{2}-m_{1}}{M} T^{\prime} R} \\
& -\frac{k}{\mu}\left(t^{\prime}+\frac{m_{2}-m_{1}}{M} t^{\prime}{ }_{R}\right)\left|\Xi\left(\tilde{\boldsymbol{x}}, t, t_{R}\right)\right|^{a b} \delta_{a c} \delta_{b d} \rho^{c} \rho^{d} .
\end{aligned}
$$

The main drawback of the Lagrangian $\tilde{\mathcal{L}}_{S U(3) \otimes U(1)}^{\star}$ is that it cannot be transformed in practice into a putative non-minimally coupled Lagrangian $\mathcal{L}_{S U(3) \otimes U(1)}^{\star}$, expressed 
in terms of the original configurational variables $\left[\boldsymbol{x}_{1}, t_{1} ; \boldsymbol{x}_{2}, t_{2}\right]$. Indeed, we would have to perform a velocity-dependent change of coordinates, which would be the pull-back of the canonical transformation (7.5).

\section{Gauging the maximal symmetry group in the two-time theory}

In terms of the canonical basis $\left[t_{a}(\tau), t_{a}^{\prime}(\tau), \tilde{\boldsymbol{x}}^{i}(\tau), \tilde{\boldsymbol{x}}^{i}(\tau), \boldsymbol{\rho}(\tau), \boldsymbol{\rho}^{\prime}(\tau)\right]$, the generator of the gauge transformations of the maximal symmetry group localized at the effective center-of-mass $\tilde{\boldsymbol{x}}$ is given by:

$$
\tilde{\bar{G}}_{\mathcal{H}}=\epsilon(t) E+\varepsilon^{i}(\tilde{\boldsymbol{x}}, t) P_{i}+\omega^{i}(\tilde{\boldsymbol{x}}, t) \bar{J}_{i}+v^{i}(\tilde{\boldsymbol{x}}, t) \bar{K}_{i}+\theta^{a}(\tilde{\boldsymbol{x}}, t) \bar{S}_{a}+\xi^{a b}(\tilde{\boldsymbol{x}}, t) \bar{N}_{a b}
$$

Defining, as in Section 4, $\tilde{\theta}^{i} \equiv \theta^{i}+\omega^{i}$ and $\tilde{\eta}^{i} \equiv \varepsilon^{i}(\tilde{\boldsymbol{x}}, t)+c_{j k}{ }^{i} \omega^{j}(\tilde{\boldsymbol{x}}, t) \tilde{x}^{j}-t v^{i}(\tilde{\boldsymbol{x}}, t)$, the transformation properties of the configurational variables are

$$
\left\{\begin{aligned}
\delta \tilde{x}^{i} & =\tilde{\eta}^{i}(\tilde{\boldsymbol{x}}, t) \\
\delta r^{a} & =c_{b c}{ }^{a} \tilde{\theta}^{b}(\tilde{\boldsymbol{x}}, t) r^{c}+\sqrt{\mu k} \xi^{a b}(\tilde{\boldsymbol{x}}, t) \pi_{b} \\
\delta t & =-\varepsilon(t) \\
\delta t_{R} & =0 .
\end{aligned}\right.
$$

Clearly, with respect to the transformations generated by $\tilde{\bar{G}}_{\mathcal{H}}$ we have:

$$
\delta^{\mathcal{H}}\left(\delta^{i j} \pi_{i} \pi_{j}+k \delta_{i j} \rho_{i} \rho_{j}\right)=0,
$$

and, since we have also $\bar{\delta}^{\prime} \tilde{E}_{R}=0$, we obtain

$$
\bar{\delta}^{\prime} \tilde{\bar{\chi}}_{-}=\bar{\delta}^{\prime}\left[\tilde{E}_{R}-\frac{m_{1}-m_{2}}{2 M \mu}\left(\delta^{i j} \pi_{i} \pi_{j}+k \delta_{i j} \rho_{i} \rho_{j}\right)\right]=0 .
$$

We conclude that, because of its invariance, this constraint does not couple to external fields. As to the constraints $\tilde{\bar{\chi}}_{+}$, the only quantities with non vanishing variation are

$$
\begin{aligned}
\delta^{\mathcal{H}} P_{i} & =-\frac{\partial \tilde{\eta}^{j}}{\partial \tilde{x}^{i}} P_{j}-\frac{\partial \tilde{\theta}^{a}}{\partial \tilde{x}^{i}} \bar{S}_{a}-\frac{\partial \xi^{a b}}{\partial \tilde{x}^{i}} \bar{N}_{a b}-M \frac{\partial}{\partial \tilde{x}^{i}}\left[g_{i j} v^{i} \tilde{x}^{j}\right] \\
\delta^{\mathcal{H}} E & =-\frac{\partial \tilde{\eta}^{j}}{\partial t} P_{j}-\frac{\partial \tilde{\theta}^{a}}{\partial t} \bar{S}_{a}-\frac{\partial \xi^{a b}}{\partial t} \bar{N}_{a b}-M \frac{\partial}{\partial t}\left[g_{i j} v^{i} \tilde{x}^{j}\right]
\end{aligned}
$$

Thus, the invariance of $\tilde{\bar{\chi}}_{+}$under the transformations (3.2) can be recovered by introducing the minimal coupling

$$
\left\{\begin{aligned}
P_{i} & \mapsto \mathcal{P}_{a}=\mathbf{H}_{a}^{i}(\tilde{\boldsymbol{x}}, t)\left[P_{i}-\frac{M}{\Theta} A_{i}(\tilde{\boldsymbol{x}}, t)-B_{i}^{(b)}(\tilde{\boldsymbol{x}}, t) S_{b}-B_{i}^{(c d)}(\tilde{\boldsymbol{x}}, t) N_{c d}\right] \\
E & \mapsto \mathcal{E}=\frac{1}{\Theta}\left[E-\frac{M}{\Theta} A_{0}(\tilde{\boldsymbol{x}}, t)-B_{0}^{(a)}(\tilde{\boldsymbol{x}}, t) S_{a}-B_{0}^{(a b)}(\tilde{\boldsymbol{x}}, t) N_{a b}\right]
\end{aligned}\right.
$$

and assuming transformation rules of the gauge fields so that the condition

$$
\delta^{g} \tilde{\bar{\chi}}_{+}^{G}=\delta^{g}\left[\mathcal{E}-\frac{1}{2 M} \delta^{a b} \mathcal{P}_{a} \mathcal{P}_{b}-\frac{1}{2 \mu}\left(\delta^{a b} \pi_{a} \pi_{b}+k \delta_{a b} \rho^{a} \rho^{b}\right)\right]=0
$$

be satisfied.

The consequent transformation rule results:

$$
\hat{\delta}_{0}^{g} \Theta(t) \quad=\dot{\epsilon}(t) \Theta(t)
$$




$$
\begin{aligned}
& \hat{\delta}_{0}^{g} g_{i j}(\tilde{\boldsymbol{x}}, t)=-\frac{\partial \tilde{\eta}^{k}(\tilde{\boldsymbol{x}}, t)}{\partial \tilde{x}^{i}} g_{k j}-\frac{\partial \tilde{\eta}^{k}(\tilde{\boldsymbol{x}}, t)}{\partial \tilde{x}^{i}} g_{k j} \\
& \hat{\delta}_{0}^{g} A_{0}(\tilde{\boldsymbol{x}}, t)=2 \dot{\varepsilon} A_{0}-A_{i} \frac{\partial \tilde{\eta}^{i}}{\partial t}-\Theta \frac{\partial}{\partial t}\left[g_{i j} v^{i} \tilde{x}^{j}\right] \\
& \hat{\delta}_{0}^{g} A_{i}(\tilde{\boldsymbol{x}}, t)=\dot{\varepsilon} A_{i}-A_{j} \frac{\partial \tilde{\eta}^{j}}{\partial \tilde{x}^{i}}-g_{i j} \frac{\partial \tilde{\eta}^{j}}{\partial t}-\Theta \frac{\partial}{\partial \tilde{x}^{i}}\left[g_{j k} v^{j} \tilde{x}^{k}\right] . \\
& \hat{\delta}_{0}^{g} B_{k}^{(a)}(\tilde{\boldsymbol{x}}, t)=c_{b c}{ }^{a} \tilde{\theta}^{b}(\tilde{\boldsymbol{x}}, t) B_{k}^{(c)}(\tilde{\boldsymbol{x}}, t) \\
& +\xi^{c d}(\tilde{\boldsymbol{x}}, t)\left[c_{c e}{ }^{a} \delta_{d f} B_{k}^{(e f)}(\tilde{\boldsymbol{x}}, t)+c_{d e}{ }^{a} \delta_{c f} B_{k}^{(e f)}(\tilde{\boldsymbol{x}}, t)\right] \\
& -\frac{\partial \tilde{\eta}^{i}}{\partial \tilde{x}^{k}} B_{i}^{(a)}(\tilde{\boldsymbol{x}}, t)-\frac{\partial \tilde{\theta}^{a}(\tilde{\boldsymbol{x}}, t)}{\partial \tilde{x}^{k}} \\
& \hat{\delta}_{0}^{g} B_{0}^{(a)}(\tilde{\boldsymbol{x}}, t)=c_{b c}{ }^{a} \tilde{\theta}^{b}(\tilde{\boldsymbol{x}}, t) B_{0}^{(c)}(\tilde{\boldsymbol{x}}, t) \\
& +\xi^{c d}(\tilde{\boldsymbol{x}}, t)\left[c_{c e}{ }^{a} \delta_{d f} B_{0}^{(e f)}(\tilde{\boldsymbol{x}}, t)+c_{d e}{ }^{a} \delta_{c f} B_{0}^{(e f)}(\tilde{\boldsymbol{x}}, t)\right] \\
& +\dot{\epsilon} B_{0}^{(a)}(\tilde{\boldsymbol{x}}, t)-\frac{\partial \tilde{\eta}^{i}}{\partial t} B_{i}^{(a)}(\tilde{\boldsymbol{x}}, t)-\frac{\partial \tilde{\theta}^{a}(\tilde{\boldsymbol{x}}, t)}{\partial t} \\
& \hat{\delta}_{0}^{g} B_{k}^{(a b)}(\tilde{\boldsymbol{x}}, t)=\tilde{\theta}^{c}(\tilde{\boldsymbol{x}}, t)\left[c_{c d}{ }^{a} B_{k}^{(d b)}(\tilde{\boldsymbol{x}}, t)+c_{c d}{ }^{b} B_{k}^{(d a)}(\tilde{\boldsymbol{x}}, t)\right] \\
& +\frac{1}{4} \xi^{c d}(\tilde{\boldsymbol{x}}, t)\left[c_{e c}{ }^{a} \delta_{d}^{b}+c_{e c}^{b} \delta_{d}^{a}+c_{e d}^{a} \delta_{c}^{b}+c_{e d}{ }^{b} \delta_{c}^{a}\right] B_{k}^{(e)}(\tilde{\boldsymbol{x}}, t) \\
& -\frac{\partial \tilde{\eta}^{i}}{\partial \tilde{x}^{k}} B_{i}^{(a b)}(\tilde{\boldsymbol{x}}, t)-\frac{\partial \xi^{a b}(\tilde{\boldsymbol{x}}, t)}{\partial \tilde{x}^{k}} \\
& \hat{\delta}_{0}^{g} B_{0}^{(a b)}(\tilde{\boldsymbol{x}}, t)=\tilde{\theta}^{c}(\tilde{\boldsymbol{x}}, t)\left[c_{c d}{ }^{a} B_{0}^{(d b)}(\tilde{\boldsymbol{x}}, t)+c_{c d}{ }^{b} B_{0}^{(d a)}(\tilde{\boldsymbol{x}}, t)\right] \\
& +\frac{1}{4} \xi^{c d}(\tilde{\boldsymbol{x}}, t)\left[c_{e c}{ }^{a} \delta_{d}^{b}+c_{e c}{ }^{b} \delta_{d}^{a}+c_{e d}{ }^{a} \delta_{c}^{b}+c_{e d}{ }^{b} \delta_{c}^{a}\right] B_{0}^{(e)}(\tilde{\boldsymbol{x}}, t) \\
& +\dot{\epsilon} B_{0}^{(a b)}(\tilde{\boldsymbol{x}}, t)-\frac{\partial \tilde{\eta}^{i}}{\partial t} B_{i}^{(a b)}(\tilde{\boldsymbol{x}}, t)-\frac{\partial \xi^{a b}(\tilde{\boldsymbol{x}}, t)}{\partial t} .
\end{aligned}
$$

Moreover, it can be checked that the integrability condition of the multi-temporal formalism, $\left\{\chi_{+}^{\mathcal{H}}, \chi_{-}^{\mathcal{H}}\right\}=0$, still holds.

Using the definitions (7.10) of Section $\mathbf{7}$, in which the time reparametrization field $\Theta(t)$ must now be introduced, i.e.:

$$
\begin{aligned}
& D \rho^{a} \quad=\rho^{a}+c_{b c}{ }^{a}\left(\tilde{x}^{\prime k} B_{k}^{(b)}(\tilde{\boldsymbol{x}}, t)+t^{\prime} B_{0}^{(b)}(\tilde{\boldsymbol{x}}, t)\right) \rho^{c} \\
& \tilde{\Xi}^{a b}\left(\tilde{\boldsymbol{x}}, t, t_{R}\right)=\delta^{a b}+\frac{\mu}{\sqrt{\mu k}} \frac{1}{\Theta(t) t^{\prime}+\frac{m_{2}-m_{1}}{M} t^{\prime}{ }_{R}}\left(\tilde{x}^{\prime k} B_{k}^{(a b)}(\tilde{\boldsymbol{x}}, t)+t^{\prime} B_{0}^{(a b)}(\tilde{\boldsymbol{x}}, t)\right),
\end{aligned}
$$


we get the final result, as a functional of the variables $\left[\tilde{\boldsymbol{x}}, \boldsymbol{\rho}, t, t_{R}\right]$, in the form:

$$
\begin{aligned}
\tilde{\mathcal{L}}_{\mathcal{H}}^{\star}= & \frac{M}{2} \frac{g_{i j}(\tilde{\boldsymbol{x}}, t) \tilde{x}^{i} \tilde{x}^{\prime j}}{\Theta(t) t^{\prime}}+M A_{i}(\tilde{\boldsymbol{x}}, t) \tilde{x}^{\prime i}+M \Theta(t) A_{0}(\tilde{\boldsymbol{x}}, t) t^{\prime} \\
& +\frac{\mu}{2} \frac{\left.\Xi^{-1}\left(\tilde{\boldsymbol{x}}, t, t_{R}\right)\right|_{a b} D \rho^{a} D \rho^{b}}{\Theta(t) t^{\prime}+\frac{m_{2}-m_{1}}{M} T^{\prime}{ }_{R}} \\
& -\frac{k}{\mu}\left(\Theta(t) t^{\prime}+\frac{m_{2}-m_{1}}{M} t^{\prime}\right)\left|\Xi\left(\tilde{\boldsymbol{x}}, t, t_{R}\right)\right|^{a b} \delta_{a c} \delta_{b d} \rho^{c} \rho^{d} .
\end{aligned}
$$

Clearly, this new Lagrangian reduces to the Lagrangian $\mathcal{L}_{\mathcal{H}}$ (5.4) when $t=\tau, t_{R}=0$. The dynamical symmetry transformations, under which this Lagrangian is invariant (quasi-invariant) are given in configuration space by:

$$
\left\{\begin{array}{l}
\delta^{\mathcal{H}} \tilde{x}^{i}=\tilde{\eta}^{i}(\tilde{\boldsymbol{x}}, t) \\
\delta^{\mathcal{H}} \rho^{a}=\epsilon(t) \dot{r}^{a}+c_{b c}{ }^{a} \tilde{\theta}^{b}(\tilde{\boldsymbol{x}}, t) \rho^{c}+\frac{\mu}{\sqrt{\mu k}} \xi^{a b}(\tilde{\boldsymbol{x}}, t) \frac{\left|\Xi^{-1}\right|_{b c}\left(\tilde{\boldsymbol{x}}, t, t_{R}\right) D \rho^{c}}{\Theta(t) t^{\prime}+\frac{m_{2}-m_{1}}{M} t_{R}^{\prime}} .
\end{array}\right.
$$

\section{Conclusions}

The main result of this paper is a well-defined algorithm for characterizing the coupling of extended, bounded, non-relativistic systems (whose binding is given by long range forces or confining interactions), having a given maximal dynamical symmetry group (semi-direct product of the Galilei group times the internal maximal dynamical symmetry group), to external gravitational and Yang-Mills fields. This algorithm, based on a generalization of Utiyama's procedure, has been applied to the nonrelativistic oscillator with center-of-mass. The explicit form of the coupling in this system is given by the Lagrangian (5.4). Its expression in terms of the coordinates of the individual constituents (see eqs. 5.9,5.10) shows the non-local nature of the coupling to the latter, since the algebraic approch privileges a local coupling to the center-of-mass and relative variables. We cannot exclude the existence of a local coupling to the constituent particles (like the standard coupling to the free test particles of a dust) but certain it cannot be obtained by exploiting algebraic methods à la Utiyama.

It seems reasonable that the method we are proposing is sensible for bound systems but not for a dust of free constituents (whose maximal symmetry group is the semidirect product of the Galilei group times $U(1)$, times $E(3)_{1}, \ldots, E(3)_{N-1}$, for $N$ particles) because, in this case, one would get a non standard coupling to the center-of-mass, contrary to the conventional wisdom about the treatment of a dust in general relativity or Galilean gravity.

Our approch allows the treatment of a dust of bounded systems by exploiting the algebraic approch for the latter and the standard approch for a dust of center-ofmasses.

We have discused also the two-time reformulation of the Galilean harmonic oscillator in which the system is described only by two first-class constraints (the canonical Hamiltonian vanishes) as a preliminary step towards the relativistic case in which only this kind of Hamiltonian description is available [8, 9]. In the relativistic case, in which the Poincaré kinematical group is present by definition - both in the 
case of a bilocal system $\dagger$ and for continuous systems as the Nambu-Goto string $\ddagger-$ the resulting "maximal symmetry algebra", including both kinematical and dynamical symmetries, is not a Lie algebra but some kind of generalized W-algebra with structure constants depending on the total four-momentum.

\section{Acknowledgments}

Roberto De Pietri wishes to thank C. Rovelli and E.T. Newman for the hospitality kindly offered to him at the Department of Physics and Astronomy. Massimo Pauri would like to express his deep appreciation and thanks to the Center for Philosophy of Science, for the warm and stimulating intellectual atmosphere experienced there and the generous partial support obtained during the completion of the present work at the University of Pittsburgh.

\section{Appendix A. Canonical realizations of the $U(3)=S U(3) \otimes U(1)$ group}

In standard notations, the canonical generators of the $U(3)=S U(3) \otimes U(1)$ group can be written as:

$$
X_{a-b}=N_{a b}+\frac{i}{2} S_{a b}
$$

(where $N_{a b}, S_{a b}$ are symmetric and antisymmetric real quantities, respectively) which satisfy the (Poisson brackets) commutation rules:

$$
\left\{X_{a-b}, X_{c-d}\right\}=i\left(\delta_{b c} X_{a-d}-\delta_{a d} X_{c-b}\right) .
$$

Expressing $N_{a b}$ in terms of its trace and traceless parts $\left(N, \tilde{N}_{a b}\right)$ as: $N_{a b}=$ $\tilde{N}_{a b}+\frac{1}{3} \delta_{a b} N$, the commutation rules can be rewritten in terms of $S_{a}, \tilde{N}_{a b}$ and $N$ as:

$$
\begin{array}{ll}
\left\{N, S_{b}\right\} & =0 \\
\left\{N, \tilde{N}_{c d}\right\} & =0 \\
\left\{S_{a}, S_{b}\right\} & =\epsilon_{a b c} S_{c} \\
\left\{S_{a}, \tilde{N}_{c d}\right\} & =\epsilon_{a c b} \tilde{N}_{b d}+\epsilon_{a d b} \tilde{N}_{c b} \\
\left\{\tilde{N}_{a b}, \tilde{N}_{c d}\right\} & =\frac{1}{4}\left[\delta_{b d} \epsilon_{a c e}+\delta_{b c} \epsilon_{a d e}+\delta_{a d} \epsilon_{b c e}+\delta_{a c} \epsilon_{b d e}\right] S_{e}
\end{array}
$$

where the $S U(3) \otimes U(1)$ group structure is explicitly evidentiated.

According to the general theory [6], a canonical realization of a Lie group $G_{r}$ (of order $r$ ) can be characterized in terms of two basic schemes: A) the scheme $A$ which depends entirely on the structure of the Lie algebra $\mathcal{L}_{G_{r}}$ (including its cohomology) and amounts to a pseudo-canonization of the generators, in terms of $k$ invariants and $h=(r-k) / 2$ pairs of canonical variables (kernel of the scheme); B) the scheme $B$ (or typical form) which is an array of $2 n$ canonical variables $P_{i}, Q_{i}$, defined by means of a canonical completion of the scheme A. The scheme $B$ allows to analyze any given canonical realization of $G_{r}$ and to construct the most general canonical realization of $G_{r}$. In what follows we shall be interested in the analysis of given realizations in

$\dagger$ The relativistic oscillator still possess a $S U(3) \otimes U(1)$ algebra of constants of motion.

$\ddagger$ See the infinite-dimensional algebra of constants of motion of reference 10. 
which the explicit expressions of the generators is already given in terms of the physical variables $p_{i}, q_{i}$ (see later).

The scheme $A$ for the group $S U(3) \otimes U(1)$ has the generic form $(k=3, h=3)$ :

\begin{tabular}{||lll|ll|l||}
\hline \hline $\mathcal{P}_{1}$ & $\mathcal{P}_{2}$ & $\mathcal{P}_{3}$ & $\mathcal{J}_{1}$ & $\mathcal{J}_{2}$ & $\mathcal{J}_{3}$ \\
$\mathcal{Q}_{1}$ & $\mathcal{Q}_{2}$ & $\mathcal{Q}_{3}$ & & & \\
\hline \hline
\end{tabular}

where variables belonging to the same vertical pair are canonical conjugated, and variables belonging to different vertical lines commute. The quantities $\mathcal{J}_{1}$, $\mathcal{J}_{2}$ and $\mathcal{J}_{3}$ clearly commute with all of the generators and are the invariants of the group. Of course, any set of $k$ functional independent functions $\mathcal{J}_{1}^{\prime}\left(\mathcal{J}_{1}, \cdots, \mathcal{J}_{k}\right), \cdots, \mathcal{J}_{k}^{\prime}\left(\mathcal{J}_{1}, \cdots, \mathcal{J}_{k}\right)$ of the invariants are good invariants as well. The explicit form of the variables (A.4), as functions of the generators, can be chosen as follows:

$$
\begin{aligned}
\mathcal{P}_{1} & =S_{z} & \mathcal{P}_{2} & =\sqrt{\vec{S}^{2}} \\
\mathcal{Q}_{1} & =\arctan \frac{S_{y}}{S_{x}} & \mathcal{Q}_{2} & =\arctan \frac{S(\vec{S} \wedge \vec{W})_{z}}{(\vec{S} \cdot \vec{W}) S_{z}-S^{2} W_{z}},
\end{aligned}
$$

where $W_{a}=\tilde{N}_{a b} S^{b}$, while $\mathcal{P}_{3}$ and $\mathcal{Q}_{3}$ are (elliptic) rotational scalar functions of their arguments:

$$
\begin{aligned}
& \mathcal{P}_{3}=\mathcal{P}_{3}\left[\vec{W} \cdot \vec{S}, \vec{W} \cdot \vec{W}, \mathcal{J}_{1}, \mathcal{J}_{2}, \mathcal{J}_{3}\right] \\
& \mathcal{Q}_{3}=\mathcal{Q}_{3}\left[\vec{W} \cdot \vec{S}, \vec{W} \cdot \vec{W}, \mathcal{J}_{1}, \mathcal{J}_{2}, \mathcal{J}_{3}\right],
\end{aligned}
$$

whose explicit expression does not matter here (see ref. [7]).

A possible choice of three independent functions of the invariants is:

$$
\begin{aligned}
& \mathcal{J}_{1}=\frac{1}{2} \sum_{a=1}^{3} S_{a}^{2}+\sum_{a, b=1}^{3} \tilde{N}_{a b} \tilde{N}_{b a} \\
& \mathcal{J}_{2}=\sum_{a, b, c=1}^{3} \tilde{N}_{a b} \tilde{N}_{b c} \tilde{N}_{c a}+\frac{3}{4} \sum_{a, b=1}^{3} \tilde{N}_{a b} S^{a} S^{b} \\
& \mathcal{J}_{3}=N
\end{aligned}
$$

A scheme $A$ is called singular [6] if, due to some functional relations, already existing or imposed, among the invariants, some canonical pairs become singular functions of the generators and must be omitted from scheme $A$ itself which, then, has to be redetermined from the beginning. In this case, the number of canonical pairs may be $m<h$.

A singular scheme $A$ for $S U(3) \otimes U(1)$ corresponds, for example, to the following conditions on the invariants:

$$
\begin{aligned}
& \mathcal{J}_{1}^{\prime} \equiv F_{1}\left(\mathcal{J}_{1}, \mathcal{J}_{2}, \mathcal{J}_{3}\right)=\mathcal{J}_{1}-\frac{2}{3} \mathcal{J}_{3}^{2}=0 \\
& \mathcal{J}_{2}^{\prime} \equiv F_{2}\left(\mathcal{J}_{1}, \mathcal{J}_{2}, \mathcal{J}_{3}\right)=\mathcal{J}_{2}-\frac{2}{9} \mathcal{J}_{3}^{3}=0
\end{aligned}
$$


which imply $m=2\left(\mathcal{Q}_{3}\right.$ and $\mathcal{P}_{3}$ missing), and $W_{a}=0$. Upon these conditions, the expression of $\mathcal{Q}_{2}$ in (A.5) loses its meaning and has to be redefined. The new expression is

$\mathcal{Q}_{2}^{\prime}=-\frac{1}{2} \arctan \frac{2 S^{2} N_{z z}-\mathcal{J}_{3}\left(S^{2}-S_{z}^{2}\right)}{S \sqrt{-\left(S^{2}-S_{z}^{2}\right)^{2}+4 \mathcal{E} N_{z z}\left(S^{2}-S_{z}^{2}\right)-4 S^{2} N_{z z}^{2}}}$.

The scheme B, i.e. the canonical completion of scheme $A$, is accomplished in general according to one of the following possibilities:

Type 1) no new variable is added to the scheme $A$ and $k$ independent function of the canonical invariants are put identically equal to constants. $2 h$ typical variables $P_{i}, Q_{j}$ are identified to the variables of the kernel. The explicit expression of the canonical generators, in terms of the variables $P_{i}, Q_{j}$ of the scheme $B$, is obtained by inverting the functions of the scheme $A$. Then, an arbitrary fixed (with respect to the group parameters) canonical transformation $\mathcal{S}$ gives the expression of the generators in terms of $2 h$ generic canonical variable $p_{i}, q_{j}$ (in our cases, these are the already given expressions of the generators in terms of the physical variables. Since the phase space contains no submanifolds invariant under the action of $G_{r}$, these realizations are called irreducible, for any $k$-uple of allowed constant values of the functions of the invariants.

Type 2) a certain number $l(l \leq k)$ of canonical variables $Q_{s}$, (called supplementary variables) turn out to be coupled, or are axiomatically coupled, to $l$ of the invariants $\mathcal{J}_{i}$, building up $l$ new canonical pairs, while $h=k-l$ independent functions of the invariants are put identically equal to constants. Then the generators, as functions of the typical variables $P_{i}, Q_{i}, Q_{s}(i, j=1, \ldots, h \quad s=1, \ldots, l)$ are obtained by inversion as before but do not depend on the supplementary variables $Q_{s}$. The phase-space is $2 h+2 l$ dimensional and, containing the submanifolds $\mathcal{J}_{t}(p, q)=$ const. $(t=1, \cdots, k-l)$ as invariant submanifolds, corresponds to non-irreducible realizations for any $(k-l)$-uple of allowed constant values of the invariant functions that have been constrained. An arbitrary fixed (with respect to the group parameters) canonical transformation $\mathcal{S}$ gives the expression of the generators in terms of $2 h+2 l$ generic canonical variables $p_{i}, q_{j}$ (in the cases discussed in the present paper, this functional dependence is the starting point, and is defined by the already given expressions of the generators in terms of the physical variables).

A particular case of Type $\mathbf{2}$ is the so-called complete realization, corresponding to $l=k$ and $(r+k) / 2$ canonical pairs. This realization is completely determined, locally, by the group structure. In geometrical terms, the variables of the the kernel together with the $k$ supplementary variables define a local chart on the orbits of the co-adjoint representation of $G_{r}$.

Type 3) an arbitrary number $v \leq n-h$ of pairs of canonical variables $Q_{u}, P_{u}$ $(u=1, \cdots, v)$ (inessential variables) turn out to exist, or are axiomatically added, to the scheme $A$. These variables are not canonically coupled to invariants nor share any functional relation with the variables of scheme $A$, so that they commute with all the variables considered up to now. Then, one proceeds, as for Type $\mathbf{1}$ or Type $\mathbf{2}$, by inverting the functional dependence of the typical variables on the generators and performing an arbitrary fixed canonical transformation which leads to the generic form of the realization in terms of $2 n$ canonical variables $p_{i}, q_{i}$. Since the inessential variables define (trivial) invariant submanifolds in phase-space, these realizations are non-irreducible. Note that Types $\mathbf{1}$-and-3, or $\mathbf{2}$-and-3, are mutually compatible. 
The canonical realization of the $S U(3) \otimes U(1)$ group, corresponding to the threedimensional isotropic harmonic oscillator, is characterized by a scheme $B$ which is a canonical completion of type $2(m=2, \quad l=1)$ of the singular scheme $A$ defined by conditions (A.8). Precisely, we have:

$$
\begin{array}{||cc|c||}
\hline \hline P_{1}(\vec{r}, \vec{\pi})=S_{z} & P_{2}(\vec{r}, \vec{\pi})=\sqrt{\vec{S}^{2}} & P_{3}(\vec{r}, \vec{\pi})=\mathcal{J}_{3}=\mathcal{E} / k \\
Q_{1}(\vec{r}, \vec{\pi})=\arctan \frac{S_{y}}{S_{x}} & Q_{2}(\vec{r}, \vec{\pi})=\mathcal{Q}_{2}^{\prime} & Q_{3}(\vec{r}, \vec{\pi})=\mathcal{Q}_{\mathcal{E}} \\
\hline \hline
\end{array}
$$

$$
\mid \mathcal{J}_{1}^{\prime} \equiv \mathcal{F}_{1}=0 \quad \mathcal{J}_{2}^{\prime} \equiv \mathcal{F}_{2}=0
$$

where:

a)

$$
\begin{aligned}
Q_{2}(\vec{\pi}, \vec{r})=\mathcal{Q}_{2}^{\prime} & \equiv-\frac{1}{2} \arctan \frac{2 S^{2} N_{z z}-\mathcal{J}_{3}\left(S^{2}-S_{z}^{2}\right)}{S \sqrt{-\left(S^{2}-S_{z}^{2}\right)^{2}+4 \mathcal{E} N_{z z}\left(S^{2}-S_{z}^{2}\right)-4 S^{2} N_{z z}^{2}}} \\
& =\frac{1}{2 s} \arctan \left[\frac{p_{\theta} \tan \theta}{s}\right]+\frac{1}{4 s} \arctan \left[\frac{s^{2}-\mathcal{E} \mu r^{2}}{s \sqrt{2 \mathcal{E} \mu r^{2}-k \mu r^{4}-s^{2}}}\right],
\end{aligned}
$$

b) the supplementary variable $Q_{3}(\vec{\pi}, \vec{r})$ has the expression

$$
Q_{3}(\vec{\pi}, \vec{r}) \equiv \mathcal{Q}_{\mathcal{E}}=\frac{1}{2} \sqrt{\frac{\mu}{k}} \arctan \left[\sqrt{\frac{\mu}{k}} \frac{\mathcal{E}-k r^{2}}{\sqrt{\mu\left(2 \mathcal{E} r^{2}-k r^{4}\right)-s^{2}}}\right],
$$

and

c) no inessential variables are present.

This realization is clearly non-irreducible, due to the fact that the internal energy $\mathcal{E}$ is an invariant which has not a fixed value. The above expression of $\mathcal{Q}_{\mathcal{E}}$ has been determined by solving the time independent Hamilton-Jacobi equation, with $S_{z}, \sqrt{\vec{S}^{2}}$ and $\mathcal{E}$ assumed as integration constants.

\section{Appendix B. Canonical Realization of the Centrally Extended Galilei Group $\mathcal{G}_{M}$}

The commutation relations of the centrally extended Galilei Lie algebra are:

$$
\left\{\begin{array}{l}
\left\{\bar{J}_{i}, J_{j}\right\}=\epsilon_{i j k} \bar{J}_{k} \\
\left\{P_{i}, J_{j}\right\}=\epsilon_{i j k} P_{k} \\
\left\{P_{i}, P_{j}\right\}=0 \\
\left\{K_{i}, J_{j}\right\}=\epsilon_{i j k} \bar{K}_{k} \\
\left\{K_{i}, K_{j}\right\}=0 \\
\left\{\bar{H}, K_{i}\right\}=-P_{i} \\
\left\{P_{i}, K_{j}\right\}=-M \delta_{i j} .
\end{array}\right.
$$


The scheme $A$ for the centrally extended Galilei group can be written:

$$
\begin{array}{||cc|cc||}
\hline \hline \mathcal{P}_{i}=P_{i} & \mathcal{P}_{4}=\Sigma_{z} & \mathcal{J}_{1}=\sqrt{\vec{\Sigma}^{2}} & \mathcal{J}_{2}=H-\frac{P^{2}}{2 M} \\
\mathcal{Q}_{i}=\frac{K_{i}}{M} & \mathcal{Q}_{4}=\arctan \frac{\Sigma_{y}}{\Sigma_{x}} & & \\
\hline \hline
\end{array}
$$

where $\vec{\Sigma}=\vec{J}-\frac{1}{M} \vec{K} \wedge \vec{P}$.

A singular scheme $A$ for the centrally extended Galilei group obtains if $\mathcal{J}_{1}=0$, which leads to $\vec{\Sigma}=0$, and therefore to the reduced scheme A:

$$
\begin{array}{|c|c|}
\hline \hline \mathcal{P}_{i}=P_{i} & \mathcal{J}_{2}=H-\frac{P^{2}}{2 M} \\
\mathcal{Q}_{i}=\frac{K_{i}}{M} & \\
\hline \hline
\end{array}
$$

\section{Appendix C. Canonical Realizations of the group $\mathcal{H}=\mathcal{G}_{M} \wedge(S U(3) \otimes U(1))$}

The scheme $A$ for the direct product $\mathcal{G}_{M} \otimes S U(3) \otimes U(1)$ is, as for any other direct product, simply obtained by joining together the schemes $A$ of the factor groups. Thus:

\begin{tabular}{|c|c|c|}
\hline \multicolumn{3}{|r|}{ invariants } \\
\hline$S U(3)$ & $U(1)$ & $\mathcal{G}_{M}$ \\
\hline $\begin{array}{ll}\mathcal{J}_{1} & \mathcal{J}_{2}\end{array}$ & $\mathcal{J}_{3}$ & $\mathcal{J}_{4}=\sqrt{\vec{\Sigma}^{2}} \quad \mathcal{J}_{5}=H-\frac{P^{2}}{2 M}$ \\
\hline
\end{tabular}

\begin{tabular}{||ccc|cc||}
\hline \multicolumn{6}{|c||}{ kernel } \\
\hline & \multicolumn{1}{|c|}{$S U(3)$} & \multicolumn{2}{c||}{$\mathcal{G}_{M}$} \\
\hline $\mathcal{P}_{1}=S_{z}$ & $\mathcal{P}_{2}=\sqrt{\vec{S}^{2}}$ & $\mathcal{P}_{3}$ & $\mathcal{P}_{3+i}=P_{i}$ & $\mathcal{P}_{7}=\Sigma_{z}$ \\
$\mathcal{Q}_{1}=\arctan \frac{S_{y}}{S_{x}}$ & $\mathcal{Q}_{2}$ & $\mathcal{Q}_{3}$ & $\mathcal{Q}_{3+i}=\frac{K_{i}}{M}$ & $\mathcal{Q}_{7}=\arctan \frac{\Sigma_{y}}{\tilde{\Sigma}_{x}}$ \\
\hline \hline
\end{tabular}

Consider now the semi-direct product of the centrally extended Galilei group and the $S U(3) \otimes U(1)$ group. The order of this group is $10+8+1=19$. Its Lie algebra is given by eq.(A.3), (B.1) plus the additional Poisson brackets which express the infinitesimal action of $\overline{\mathcal{G}_{M}}$ onto the invariant subgroup $S U(3) \otimes U(1)$; precisely:

$$
\left\{\begin{array}{rl}
\left\{J_{i}, S_{a}\right\} & =\epsilon_{i a c} S_{c} \\
\left\{J_{i}, N_{a b}\right\} & =\epsilon_{i a c} N_{c b}+\epsilon_{i b c} N_{a c}
\end{array} .\right.
$$

The scheme $A$ for the group $\mathcal{H}$ can then be written: 


\begin{tabular}{||ccc|cc||}
\hline \multicolumn{5}{||c||}{ kernel } \\
\hline $\mathcal{P}_{1}=S_{z}$ & $\mathcal{P}_{2}=\sqrt{\vec{S}^{2}}$ & $\mathcal{P}_{3}$ & $\mathcal{P}_{3+i}=P_{i}$ & $\mathcal{C}$ \\
\hline \multirow{2}{*}{$\mathcal{Q}_{1}=\arctan \frac{S_{y}}{S_{x}}$} & $\mathcal{Q}_{2}$ & $\mathcal{Q}_{3}$ & $\mathcal{Q}_{3+i}=\frac{K_{i}}{M}$ & $\mathcal{Q}_{7}=\arctan \frac{\sigma_{y}}{\sigma_{x}}$ \\
\hline \hline
\end{tabular}

\begin{tabular}{|c|c|c|c|}
\hline \multicolumn{4}{|c|}{ invariants } \\
\hline$S U(3)$ & $U(1)$ & & $\mathcal{C}$ \\
\hline $\mathcal{J}_{1} \quad \mathcal{J}_{2}$ & $\mathcal{J}_{3}$ & $\mathcal{J}_{4}=\sqrt{\vec{\sigma}^{2}}$ & $\mathcal{J}_{5}=H-\frac{P^{2}}{2 M}$ \\
\hline
\end{tabular}

where $\mathcal{C}$ means the Galilei groups associated to the center-of-mass coordinates, $\mathcal{Q}_{2}$, $\mathcal{Q}_{3}, \mathcal{P}_{3}, \mathcal{J}_{1}, \mathcal{J}_{2}, \mathcal{J}_{3}$ are given by eqs. A.5.,(A.6),(A.7) and $\vec{\sigma}$ is an angular momentum vector defined by:

$$
\vec{\sigma} \equiv \vec{\Sigma}-\vec{S}=\vec{J}-\frac{1}{M} \vec{K} \wedge \vec{P}-\vec{S}
$$

so that

$$
\left\{S_{i}, \sigma_{j}\right\}=0
$$

Note that the only difference between the schemes $A$ described in (C.1) and (C.3) is precisely due to the substitution

$$
\vec{\Sigma} \Rightarrow \vec{\sigma}
$$

which accounts for the action of $\mathcal{G}_{M}$ onto $S U(3)$. The variables of the sub-scheme $\mathcal{C}$ formally correspond to a new centrally extended Galilei group.

The canonical realization of $\mathcal{H}$ corresponding to the three dimensional isotropic harmonic oscillator with center-of-mass discussed in the present paper corresponds to a singular scheme $A$ in which four of the invariant are fixed in the following way:

$$
\begin{aligned}
& \mathcal{J}_{1}^{\prime}=\mathcal{J}_{1}-\frac{2}{3} \mathcal{J}_{3}^{2}=0 \\
& \mathcal{J}_{2}^{\prime}=\mathcal{J}_{2}-\frac{2}{9} \mathcal{J}_{3}^{3}=0 \\
& \mathcal{J}_{4}=0 \quad(\text { which implies } \vec{\Sigma} \equiv \vec{S}) \\
& \mathcal{J}_{5}^{\prime}=\mathcal{J}_{5}-\sqrt{\frac{k}{\mu}} \mathcal{J}_{3}=0 .
\end{aligned}
$$

These conditions lead to $m=5\left(\mathcal{Q}_{3}, \mathcal{P}_{3}, \mathcal{Q}_{7}, \mathcal{P}_{7}\right.$ missing $)$ while $\mathcal{Q}_{2}$ has to be replaced by the expression given in eq. (A.12). In conclusion, the scheme $B$ of the realization has the form: 


\begin{tabular}{|c|c|c|}
\hline \multicolumn{3}{|c|}{ kernel } \\
\hline$\overline{S U}$ & & $\mathcal{C}$ \\
\hline $\begin{array}{c}P_{1}(\vec{r}, \vec{\pi})=S_{z} \\
Q_{1}(\vec{r}, \vec{\pi})=\arctan \frac{S_{y}}{S_{r}}\end{array}$ & $\begin{array}{c}P_{2}(\vec{r}, \vec{\pi})=\sqrt{\vec{S}^{2}} \\
Q_{2}(\vec{r}, \vec{\pi})\end{array}$ & $\begin{aligned} P_{3+i}(\vec{x}, \vec{p}) & =P_{i} \\
\mathcal{Q}_{3+i}(\vec{x}, \vec{p}) & =\frac{K_{i}}{M}\end{aligned}$ \\
\hline
\end{tabular}

\begin{tabular}{|c|c|c|}
\hline \multicolumn{3}{|c|}{ invariants } \\
\hline$S U(3)$ & $U(1)$ & $\mathcal{C}$ \\
\hline $\begin{array}{cc}\mathcal{J}_{1}^{\prime} \equiv \mathcal{F}_{1}=0 & \mathcal{J}_{2}^{\prime} \equiv \mathcal{F}_{2}=0 \\
- & -\end{array}$ & $\begin{array}{c}\mathcal{J}_{3}=\mathcal{E} / k \\
Q_{\mathcal{E}}(\vec{r}, \vec{\pi})\end{array}$ & $\begin{array}{c}\mathcal{J}_{4}=0 \quad \mathcal{J}_{5}^{\prime}=\mathcal{J}_{5}-\sqrt{\frac{k}{\mu}} \mathcal{J}_{3}=0 \\
-\end{array}$ \\
\hline
\end{tabular}

It is seen that (C.8) is a scheme $B$ of the direct product of $S U(3) \otimes U(1)$ times the singular realization of $\mathcal{C}$ corresponding to the Galilei group transformations embodied by a scalar body (center-of-mass).

Thus, the dynamical system we are describing has 11 functionally independent constants of the motion, precisely: the total momentum $(\vec{P})$, a vector $\left(\vec{R}=\frac{1}{M} \vec{K}-t \vec{P}\right)$ which expresses the inertial motion of the center-of-mass, the internal angular momentum $(\vec{S})$ (which is equal to the total "spin" of the system), the internal energy $\left(\mathcal{E} \equiv H-\frac{\vec{P}^{2}}{2 M}\right)$ and an angular parameter $\left(Q_{2}\right)$ which fixes the principal axis of the orbits. On the other hand, $\mathcal{Q}_{\mathcal{E}}$ parametrizes the evolution on the orbits.

\section{References}

[1] R. De Pietri, L. Lusanna and M. Pauri: "Standard and Generalized Newtonian Gravities as "Gauge" Theories of the Extended Galilei Group - I: the Standard Theory", Classical and Quantum Gravity 12(1995)219.

[2] R. Utiyama, "Invariant Theoretical Interpretation of Interactions", Phys. Rev. 101,(1956),1597.

[3] A. Barducci, B. Casalbuoni and L. Lusanna, "Classical spinning particles interacting with YangMills fields", Nucl. Phys. B124,(1977),93.

[4] G. Longhi and L. Lusanna: "On the many-time formulation of classical particle dynamics", J. Math. Phys. 30,8,(1989),1893.

[5] K. Pohlmeyer: "A group-theoretical approach to the quantization of the free relativistic closed string", Physics Letters, 119B,(1982),100; and K. Pohlmeyer, K.H. Rehren: "Algebraic properties of the invariant charges of the Nambu-Goto theory", Commun. Math. Phys., 105, (1986), 593

[6] M. Pauri and G.M. Prosperi: "Canonical Realizations of Lie Symmetry Groups", J. Math. Phys. 7,(1966),366; and "Canonical Realizations of the Galilei Group", J. Math. Phys. $\mathbf{9},(1968), 1146$.

[7] R. De Pietri and M. Pauri: "A Peculiar Class of Canonical Realizations of the $U(3)$ group", in preparation.

[8] Ph. Droz-Vincent, Lett.Nuovo Cim. 1 (1969) 839; 7 (1973) 206; Phys.Scripta 2 (1970) 129; Rep. Math.Phys. 8 (1975) 79; Ann.Inst.H.Poincaré 27 (1977) 407; Phys.Rev. D19 (1979) 702. I.T.Todorov, Dubna preprint 1976, JINR E2-10125 (unpublished); Ann.Inst.H.Poincaré 28A (1978) 207. V.A.Rizov, H.Sazdjian and I.T.Todorov, Ann.Phys. (N.Y.) 165 (1985) 59. A.Komar, Phys.Rev. D18 (1978) 1881, 1887 and 3017.

[9] Ph. Droz-Vincent and P. Nuvowsky, "Symmetries in Predictive Relativstic Mechanics", J. Math. Phys. 31,(1990),2393.

[10] K. Pohlmeyer, K.H. Rehren: "Algebraic properties of the invariant charges of the Nambu-Goto theory" Commun. Math. Phys. 105(1986),593; F. Colomo, G. Longhi and L. Lusanna, Int. J. Mod. Phys. A7,(1992),1705; F. Colomo, G. Longhi and L. Lusanna, Int. J. Mod. Phys. A7,(1992),4107; 